\title{
Recurrent Neural Networks and Proper Orthogonal Decomposition with Interval Data for Real-Time Predictions of Mechanised Tunnelling Processes
}

\author{
S. Freitag ${ }^{1}$, B.T. Cao ${ }^{2}$, J. Ninić ${ }^{3}$, G. Meschke ${ }^{2}$
}

\begin{abstract}
${ }^{1}$ Institute for Structural Mechanics, Ruhr University Bochum, Universitätsstr. 150, 44801 Bochum, Germany

Corresponding author. Tel.: +49-234-32-29061, Fax.: +49-234-32-14149, email: steffen.freitag@sd.rub.de

${ }^{2}$ Institute for Structural Mechanics, Ruhr University Bochum, Universitätsstr. 150, 44801 Bochum, Germany UK

${ }^{3}$ Centre for Structural Engineering and Informatics, The University of Nottingham,
\end{abstract}

\begin{abstract}
A surrogate modelling strategy for predictions of interval settlement fields in real time during machine driven construction of tunnels, accounting for uncertain geotechnical parameters in terms of intervals, is presented in the paper. Artificial Neural Network and Proper Orthogonal Decomposition approaches are combined to approximate and predict tunnelling induced time variant surface settlement fields computed by a process-oriented finite element simulation model. The surrogate models are generated, trained and tested in the design (offline) stage of a tunnel project based on finite element analyses to compute the surface settlements for selected scenarios of the tunnelling process steering parameters taking uncertain geotechnical parameters by means of possible ranges (intervals) into account. The resulting mappings of time constant geotechnical interval parameters and time variant deterministic steering parameters onto the time variant interval settlement field are solved offline by optimisation and online by interval analyses approaches using the midpoint-radius representation of interval data. During the tunnel construction, the surrogate model is designed to be used in real-time to predict interval fields of the surface settlements in each
\end{abstract}


stage of the advancement of the tunnel boring machine for selected realisations of the steering parameters to support the steering decisions of the machine driver.

Keywords: surrogate model; recurrent neural networks; proper orthogonal decomposition; interval analysis; mechanised tunnelling; real-time prediction

\section{Introduction}

Mechanised tunnelling is an efficient tunnelling technology for the construction of new underground infrastructures, in particular in urban environments. During the construction process, it is required to reduce the tunnelling induced settlements or to avoid that tolerances with respect to settlements, which eventually may result in damage of existing structures, are exceeded. The settlements can be controlled by adjusting the process parameters such as the face support pressure at the tunnel face, the grouting pressure of the grouting material injected into the gap between the tunnel lining and the surrounding soil, and the advance speed during the tunnel advancement [1]. Currently, decisions affecting the steering of tunnel boring machines (TBMs) are based upon engineering expert knowledge and monitoring data. However, using monitoring data implies that information (data) related to already passed situations is used to extrapolate on the future behaviour of the soil-tunnel interactions. In this paper, an approach is presented, in which a computational model is used to support TBM steering by providing predictions on the expected settlement field in real time during the construction. Epistemic uncertainties of the geotechnical parameters are taken into account by means of interval data.

In recent years, numerical methods, in particular the finite element method, have become a standard tool for predictions of interactions between the tunnel construction and the surrounding soil in the design phase of mechanised tunnelling projects. A large number of 3D finite element simulation models have been developed to represent the staged tunnelling process, characterised by a repeated sequence of soil excavation, advancement of the TBM and the installation of a new ring of lining segments (see $[2,3,4,5]$ and references therein). More recently, a process oriented 3D numerical model for shield-supported tunnel excavation in fully saturated soft soils has been developed in [6], taking into account the most important components of mechanised tunnelling and their mutual interactions. In this computational model, a realistic representation for the soil excavation process following arbitrary alignments is enabled by means of an adaptive re-meshing technique [7]. Using this numerical model, uncertain geotechnical conditions and the interaction of the relevant components involved in the construction process have been investigated in $[8,9]$. Considering the large computational effort for realistic tunnelling models, parallelisation techniques are addressed in [10].

Information on the geotechnical situation, such as the topology and specification of the soil layers and the soil material parameters, can only be obtained from a few 
borehole data in the design stage of a project. This uncertainty resulting from limited information has to be adequately considered, when mechanised tunnelling simulations are used as the basis for the tunnel design. Stochastic numbers can be used to quantify aleatoric uncertainty considering spatial variability of geotechnical parameters by random fields, see e.g. [11] and [12]. However, a sufficiently large number of samples is required to select an adequate stochastic model and estimate the corresponding model parameters. In geotechnical reports of tunnelling projects, however, often only a few number of samples are provided to quantify the uncertainty of soil parameters. Moreover, the correlation function and the correlation length within random field approaches must be defined, which, due to insufficient data, are often based on assumptions without physical meaning. An alternative to stochastic approaches are non-stochastic or polymorphic uncertainty models, see e.g. [13]. Non-stochastic approaches such as methods based upon ranges (intervals) of parameters are frequently used in geotechnical engineering and tunnelling in the context of rock mass classifications within the rock mass rating system [14] or by means of fuzzy models [15]. A comparison of geotechnical analysis using stochastic numbers and intervals is contained in [16]. Polymorphic uncertainty models using probability boxes (p-boxes) have been applied within a random set finite element analysis for reliability assessment of tunnel construction according to the New Austrian Tunnelling Method in [17]. A holistic concept for reliability analyses in mechanised tunnelling with polymorphic uncertain data based on stochastic, interval, fuzzy, and imprecise probability approaches is presented in [8] and applied to a mechanised tunnelling simulation with fuzzy stochastic and fuzzy soil parameters in [9].

In this paper, a non-stochastic approach based on interval data is proposed for realtime predictions of tunnelling induced surface settlements. Thereby, the ranges for expected geotechnical parameters of the soil layers given in the geotechnical reports are directly quantified as intervals without requiring any further assumptions, e.g. in terms of stochastic distributions or fuzzy membership functions.

In order to support TBM steering directly at the construction site, the simulation results are required in real-time. "Real time" means a duration for the analysis, which is significantly smaller compared to the time required for one construction stage, consisting of an excavation and machine advancement step, the segmented tunnel ring installation and the tail void grouting. The time required for one construction step depends on the geological situation and is typically in the range between 2-4 hours. Hence, a real time prediction should be performed in the range of seconds to a few minutes. Moreover, the TBM steering assistant system, which is currently developed within project $\mathrm{C} 1$ of the Collaborative Research Center SFB 837 "Interaction Modeling in Mechanised Tunnelling" aims to provide predictions for user-defined realisations of the steering parameters in a few seconds. Evidently, for such real-time predictions, surrogate models are inevitable to substitute the expensive and complex finite element simulation model in mechanised tunnelling. Different methods can be used for generating simulation based surrogate models, including regression models [18], artificial neural networks [19, 20], stochastic approaches such as Kriging or spatial correlation models [21, 22], response surface methods [23], or proper orthogonal 
decomposition (POD) [24]. A study on the performance of different surrogate modelling techniques (quadratic polynomial regression, moving least squares, POD with radial basis functions (RBF), and an extended version of POD-RBF) for application in mechanised tunnelling problems is presented in [25]. In [26], a procedure for the steering of TBMs based upon artificial neural networks used as surrogate models has been proposed. However, in this method, parameter uncertainties have not been considered.

In this paper, a hybrid surrogate modelling strategy proposed in [27] is used to support decisions related to the steering of the TBM during tunnel construction in real time, taking uncertainties of geotechnical parameters into account. The hybrid strategy is based on a combination of Recurrent Neural Networks (RNN) and Gappy Proper Orthogonal Decomposition (GPOD) methods and can predict (extrapolate) tunnelling induced uncertain time and spatially varying surface settlements. Based on RNN predictions at selected monitoring points of the settlement field, the GPOD method is used to predict the whole surface field of settlements induced by the mechanised tunnelling process. In [27], an optimisation approach based on Particle Swarm Optimisation is utilized to predict the interval bounds of tunnelling induced settlement fields, considering uncertain soil parameters by means of intervals. However, this requires one to two hours on a standard notebook or parallel runs on a computing cluster to reduce the computational time, which limits its practical application for realtime predictions at construction sites. Therefore, the objective of the new surrogate modelling approach is to provide interval surface settlement predictions in real-time, i.e. in a few seconds using a standard notebook computer. This paper is based upon [28], where the calibration of the hybrid surrogate model with monitoring data and an application for real-time predictions on a standard notebook computer has been presented. As an extension, this paper includes additional research on computing the hybrid RNN-GPOD surrogate model with interval data based on the midpoint-radius representation to directly account for uncertain geotechnical parameters when performing the analysis. The RNN and GPOD surrogate models are split for midpoint and radius computations of the interval data, respectively. While the midpoint computations are identical to the approach presented in [28], the radius computations are restricted to positive numbers. To account for this constraint, we propose using NonNegative Matrix Factorisation for the GPOD part and adequate activation functions in the RNN part of the hybrid surrogate model.

The remainder of this paper is organised as follows. Section 2 gives a brief description of the process-oriented finite element model for numerical simulations of mechanised tunnelling processes, which is used for the training of the surrogate model. In Section 3, the mapping and computation of processes with interval data is introduced for time variant settlement field predictions in mechanised tunnelling. The new RNNGPOD surrogate modelling approach for interval data is presented in Section 4. The verification with an analytical solution and an application example are finally shown in Section 5. 


\section{Finite element model for mechanised tunnelling sim- ulation}

In this paper, the process-oriented finite element simulation model ekate [29] is used for the simulation of the shield tunnelling processes in fully and partially saturated soft soils. The simulation model ekate has been developed within the object-oriented finite element framework KRATOS [30]. The finite element model considers all relevant components of the tunnel construction process (soil and groundwater conditions, tunnel lining, the TBM with shield and hydraulic jacks, tail void grouting and various types of face support) and their (time dependent) interactions, as shown in Figure 1.

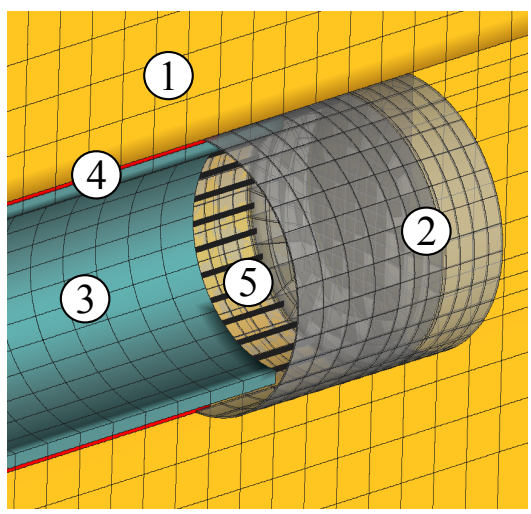

(1)
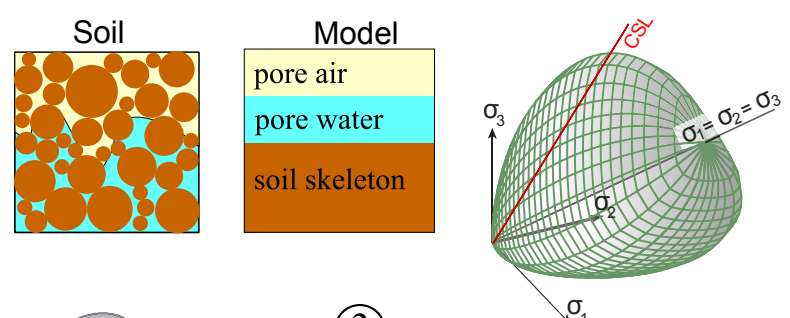

(2)
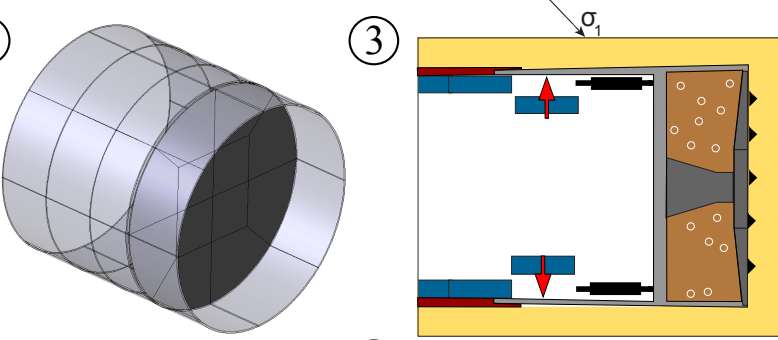

(4)

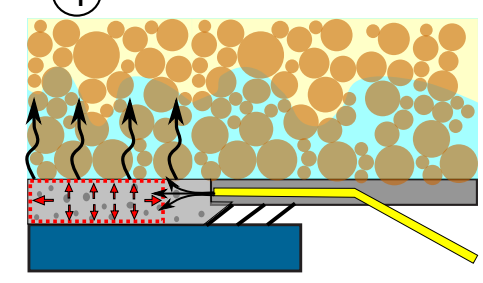

(5)

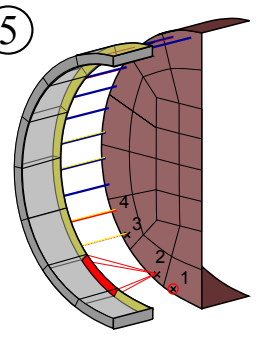

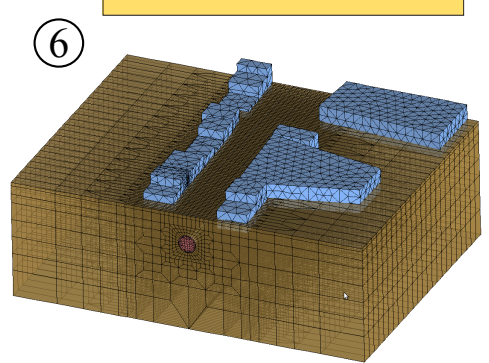

Figure 1: Components of the finite element model for shield tunnelling ekate in soft soils: (1) surrounding soil, (2) TBM, (3) segmented lining, (4) pressurisation of grouting material with time-dependent stiffness and permeability, (5) hydraulic jacks and (6) representation of existing buildings.

The soil is modelled as a three (or two) phase material for partially (or fully) saturated soils [29]. Two elastoplastic models are available for the consideration of the inelastic response of soft soils: the Clay and Sand model [31] and the Drucker-Prager model (Figure 1(1)). The TBM is represented as a deformable body moving through the soil and interacting with the ground through frictional surface-to-surface contact, allowing that the deformation of the soil naturally follows the real, tapered geometry of the TBM and that the effect of overcutting (Figure 1(2)) is captured. The hydraulic 
jacks advancing the machine are represented by truss elements tied between the lining and the shield machine (see Figure 1(5)). In order to realistically model the movement of the machine and its interaction with the soil, an algorithm to control the individual jack thrusts similar to the one proposed in [5] is used to keep the machine on the designed alignment path [7]. The load of the back-up trailer is considered by prescribing equivalent surface loads on the corresponding lining elements.

After each TBM advance, the excavation at the cutting face, the tail void grouting and the erection of a new lining ring during standstill are taken into account by re-zoning the finite element mesh and adjusting all boundary conditions to the new situation. The installation of the tunnel lining follows a staged construction process (Figure 1(3)). Within each installation step, a full lining ring of a given length, diameter and thickness is activated. The annular gap between the segmented lining tube and the excavation boundary is refilled with grout (see Figure 1(4)). The tail gap grouting is modelled as a fully saturated two-phase material with a hydrating matrix phase, considering the temporal evolution of stiffness and permeability of the cementitious grout [32]. To provide the stability of the tunnel face and to reduce ground loss behind the tapered shield, the face support pressure and the grouting pressure are applied at the tunnel face and in the steering gap, respectively.

In the simulation model ekate, the interaction of buildings including their foundations with tunnelling-induced deformations of the soil is accounted for by adopting an interface formulation (see Figure 1(6)). Buildings are described as 3D block models with a substitute stiffness computed according to [33]. In the presented finite element formulation, isotropic shell, or, alternatively, volume elements with respective properties are adopted interacting with the soil through a mesh independent surfaceto-surface contact algorithm, which prevents the penetration of the foundation of the building into the soil. It also takes into account different mechanisms of the soilstructure interaction corresponding to so-called "sagging" and "hogging" modes.

\section{Uncertainty quantification by means of intervals}

The prediction of tunnelling induced process responses, such as time variant settlements, requires to consider the unavoidable uncertainty associated with the geotechnical model. The uncertainty of geotechnical parameters describing the topology and the properties of the soil layers may have a significant influence to the time variant settlement field and, consequently, on the prognoses of tunnelling related risks. In the design stage of a tunnel project, only limited information of geotechnical parameters can be obtained from a few borehole data along the tunnel track, see Figure 2. Geotechnical reports generally provide ranges for expected geotechnical parameters, e.g. for the soil material parameters (Table 1) instead of deterministic values. The ranges for expected geotechnical parameters can directly be quantified by intervals

$$
\bar{x}=\left[{ }_{l} x,{ }_{u} x\right]
$$




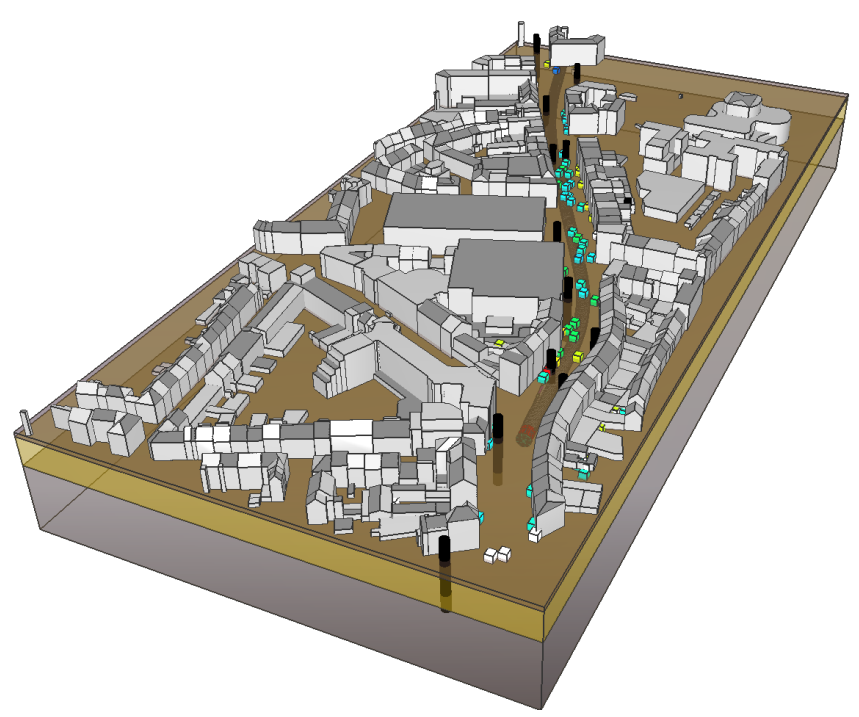

Figure 2: Digital tunnelling information model with soil layers, existing buildings, tunnel alignment, boreholes and monitoring points for the Wehrhahn-Line project in Düsseldorf according to [34].

\begin{tabular}{lr} 
Geotechnical parameter & Range \\
\hline Young's Modulus of layer $1-E_{1}[\mathrm{MPa}]$ & $10-30$ \\
Young's Modulus of layer $2-E_{2}[\mathrm{MPa}]$ & $30-90$ \\
Friction angle of layer $1-\phi_{1}\left[^{\circ}\right]$ & $25-35$ \\
Friction angle of layer $2-\phi_{2}\left[^{\circ}\right]$ & $30-40$ \\
Cohesion of layer $1-c_{1}[\mathrm{kPa}]$ & $0-3$ \\
Cohesion of layer $2-c_{2}[\mathrm{kPa}]$ & $0-3$ \\
\hline
\end{tabular}

Table 1: Ranges of exemplified soil material parameters obtained from a geotechnical report.

with lower bounds ${ }_{l} x$ and upper bounds ${ }_{u} x$. An overline is used to indicate, that a number is given as an interval. The algorithms developed in this paper are based on the midpoint-radius representation of intervals, see Figure 3, where the midpoint is

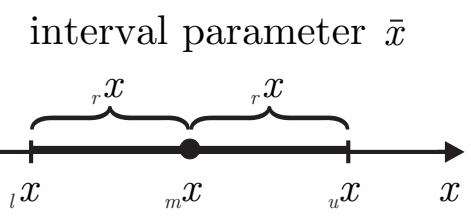

Figure 3: Interval of a geotechnical parameter $\bar{x}$, defined by its lower bound ${ }_{l} x$, upper bound ${ }_{u} x$, midpoint ${ }_{m} x$ and radius ${ }_{r} x$. 
defined by

$$
{ }_{m} x=\frac{{ }_{l} x+{ }_{u} x}{2}
$$

and the radius is given by

$$
{ }_{r} x=\frac{{ }_{u} x-{ }_{l} x}{2} .
$$

Vice versa, the lower and upper interval bounds are obtained by

$$
{ }_{l} x={ }_{m} x-{ }_{r} x
$$

and

$$
{ }_{u} x={ }_{m} x+{ }_{r} x
$$

respectively.

Considering the time dependence of mechanised tunnelling processes, the settlements at points $i=1, \ldots, I$ of an area of interest are represented as interval processes

$$
\bar{s}_{i}(t)=\left\{{ }^{[1]} \bar{s}_{i}, \ldots,{ }^{[n]} \bar{s}_{i}, \ldots,{ }^{[N]} \bar{s}_{i}\right\},
$$

which consist of a series of intervals ${ }^{[n]} \bar{s}_{i}$ with equidistant time points ${ }^{[n]} t$. All discretised surface settlements of an area of interest are summarised in the interval vector ${ }^{[n]} \overline{\mathbf{S}}$. In general, the interval vector ${ }^{[n]} \overline{\mathbf{S}}$ contains the lower and upper bounds of the nodal displacements at the surface of the finite element mesh or, alternatively, postprocessed displacements at arbitrary (mesh independent) positions.

\subsection{Mappings of processes with interval parameters in mechanised tunnelling}

The uncertainty of the time variant settlement field $\overline{\mathbf{S}}(t)$ is resulting from the uncertain geotechnical parameters collected in the interval vector $\overline{\mathbf{X}}$. During the tunnelling process, the tunnel-soil interactions, and thus the settlements, are influenced by time variant deterministic process parameters, such as the face support pressure, the grouting pressure or the advancement rate, which the machine driver controls to steer the TBM. These steering parameters are collected in the vector $\mathbf{P}(t)$. Taking deterministic steering parameters and geotechnical interval parameters into account, the following mappings are formulated:

- Type 1 Mapping with deterministic model parameters

$$
\overline{\mathbf{X}}, \mathbf{P}(t) \mapsto \overline{\mathbf{S}}(t)
$$

The (time constant) geotechnical interval parameters $\overline{\mathbf{X}}$ and the time variant deterministic steering parameters $\mathbf{P}(t)$ are defined as inputs, which are mapped with deterministic model parameters onto the time variant interval settlement field $\overline{\mathbf{S}}(t)$. 
- Type 2 Mapping with interval model parameters

$$
\mathbf{P}(t) \mapsto \overline{\mathbf{S}}(t)
$$

Only the time variant deterministic steering parameters $\mathbf{P}(t)$ are defined as inputs, which are mapped with interval model parameters onto the time variant interval settlement field $\overline{\mathbf{S}}(t)$. The influence of the time constant geotechnical interval parameters $\overline{\mathbf{X}}$ is considered by interval model parameters of the mapping model.

With respect to the mapping types for interval and fuzzy processes introduced in [35] and [36], the mapping with deterministic parameters is denoted as Type 1 Mapping and the mapping with interval parameters is denoted as Type 2 Mapping. The difference between the two mapping types lies in the way how the interval uncertainty of the geotechnical parameters $\bar{X}$ is considered. In case of Type 1 Mapping, the interval parameters $\overline{\mathbf{X}}$ are defined as additional inputs. Whereas in Type 2 Mapping, the interval parameters are fixed and only the deterministic steering parameters $\mathbf{P}(t)$ are considered as inputs. Hence, the input dimension is reduced in case of Type 2 Mapping. The algorithms developed in this paper are based on Type 1 Mapping (Eq. (7)), but they can also be applied to Type 2 Mapping with some minor modifications. For tunnelling induced settlement predictions, multiple (but low dimensional) input processes (i.e. a few $j=1, \ldots, J$ time constant geotechnical interval parameters $\bar{x}_{j}$ of the interval vector $\overline{\mathbf{X}}$ and not more than $K=3$ deterministic steering parameters $p_{k}(t), k=1, \ldots, K$, of the vector $\left.\mathbf{P}(t)\right)$ are mapped onto multiple (high dimensional) outputs (i.e. all $i=1, \ldots, I$ components $\bar{s}_{i}(t)$ of the interval settlement field summarised in the interval vector $\overline{\mathbf{S}}(t)$, where the number of settlement field components to be predicted is $I \approx 100$ ). With respect to the representation of interval processes in Eq. (6), the interval settlements ${ }^{[n]} \bar{s}_{i}$ of time step $n$ depend on the $k=1, \ldots, K$ deterministic steering parameters ${ }^{[r]} p_{k}$ of prior and current time steps $r=1, \ldots, n$, i.e. the current settlements are dependent on the history of the steering parameters adopted during the advancement process.

\subsection{Computational strategies for mappings with interval processes}

For the described mappings of processes with interval parameters, three computational approaches are possible, see Figure 4:

1. optimisation with a deterministic mapping model (for Type 1 Mapping),

2. interval arithmetic (for Type 1 and Type 2 Mapping) and

3. splitting into independent deterministic mapping models for midpoints and radiuses (for Type 1 and Type 2 Mapping).

Within optimisation approaches, see e.g. [37], the lower and upper interval bounds $\left({ }_{l}^{[n]} s_{i}\right.$ and $\left.{ }_{u}^{[n]} s_{i}\right)$ of each settlement field component have to be computed by solving 


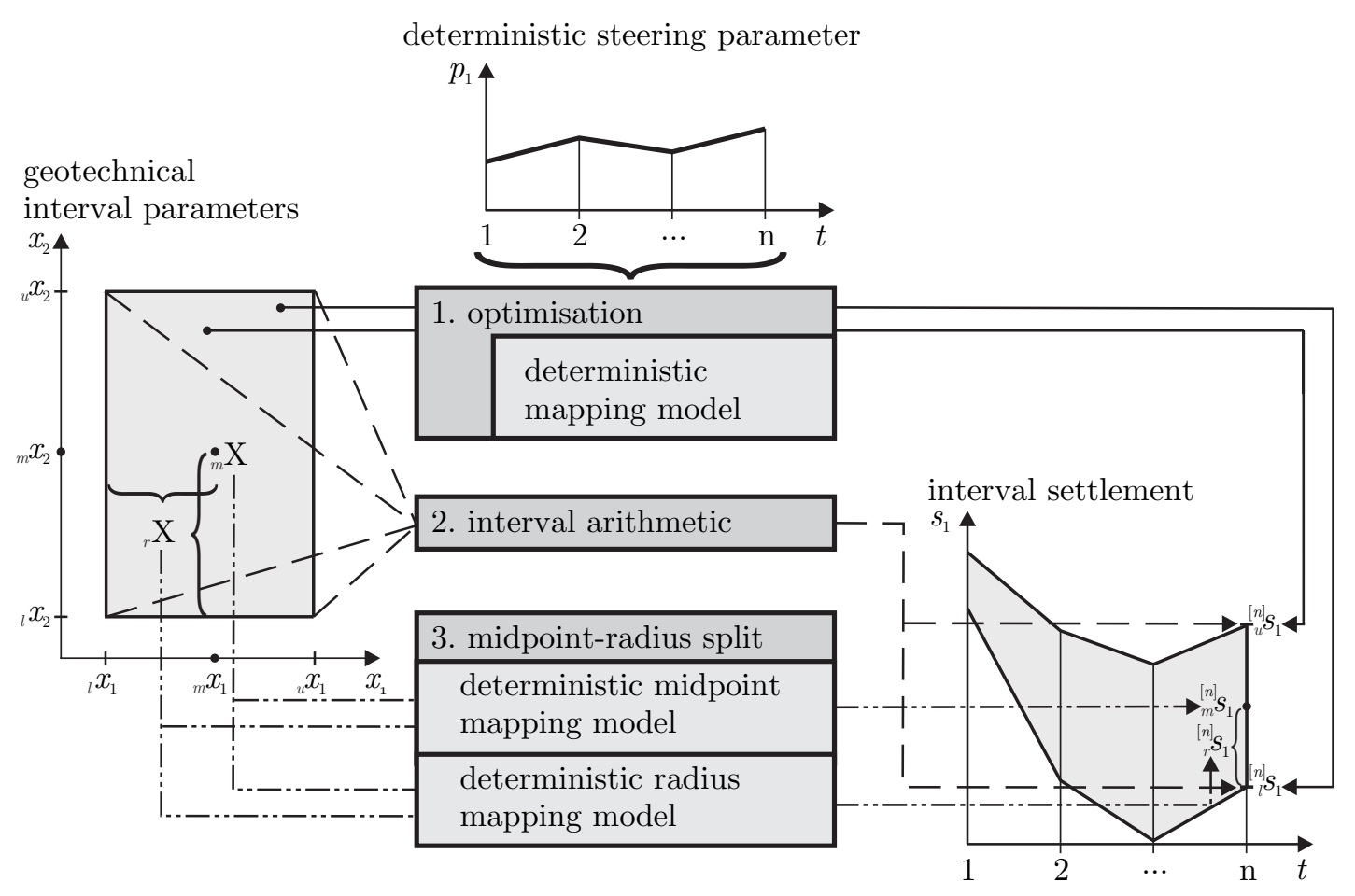

Figure 4: Computational strategies for the mapping of deterministic steering parameters and geotechnical interval parameters onto interval settlements.

two optimisation tasks (minimisation for lower bounds and maximisation for upper bounds). This might be very time consuming for high dimensional multiple output mappings, because for each settlement field component multiple runs of a deterministic mapping model have to be performed. In [27], the Particle Swarm Optimisation method in combination with a hybrid RNN-GPOD surrogate model is utilized to compute the interval bounds of 105 settlement field components during a tunnelling process. For each time step, the computational time for solving the corresponding optimisation problems was approximately 1.5 hours using a standard notebook computer. Also if the optimisation runs may be parallelised, faster approaches are required for real-time applications.

Interval arithmetic approaches, e.g. interval analysis [38], are very efficient with respect to computational time, because the whole settlement field can be computed in one computational step, without solving optimisation problems. However, if a deterministic mapping model is used to process interval data with interval arithmetic operations, the interval width of the settlement field components may be too wide, i.e. overestimated. The problem of possible overestimation has to be considered within the creation of the mapping model. For artificial neural network based mapping models, overestimation is avoided by special training algorithms for interval data, similar to the approach presented in [36] for fuzzy data. For POD-RBF in general it is possible to apply interval arithmetic operations, but overestimation cannot be avoided. In case of a black box solver for the structural model, interval arithmetic cannot directly 
be applied. First, an optimization based approach is employed to compute the interval output fields for given interval inputs. Then an interval surrogate model, e.g. an artificial neural network or a POD-RBF model, has to be created to map the interval inputs onto interval outputs by means of interval arithmetic.

In this paper, the third option is used to enable real-time predictions of interval settlement fields by means of the midpoint-radius representation of intervals. The mapping is realised by a split into independent deterministic mapping models for the midpoints and the radiuses. It should be noted, that it may also be possible to define two mapping models for the lower and upper interval bounds, which are, however, not independent from each other, because the lower bound of the output must be greater than the upper bound. The advantage of the midpoint-radius representation is that the two corresponding mapping models are independent from each other. For the mapping model for the radiuses one has to account for the constraint that only positive numbers are possible. For real-time predictions, numerically efficient surrogate models based on artificial neural networks and proper orthogonal decomposition are utilized for the mappings of midpoints and radiuses. These methods are described in the next sections.

\section{Surrogate models for predicting time variant inter- val settlement fields}

Mechanised tunnelling simulations using the process oriented finite element model presented in Section 2 are time consuming and are therefore best used in the design stage of a tunnelling project. For a model-based support of steering decisions during the tunnel construction in real-time, the finite element simulation model is replaced by surrogate models to describe the dependencies between the inputs (interval geotechnical parameters and deterministic steering parameters) and the corresponding outputs (time variant surface settlements). Several surrogate modelling approaches have been developed for various engineering applications, see e.g. [39] for an overview. Each surrogate modelling approach has specific advantages for specific tasks. As an example, Artificial Neural Networks are advantageous for multiple input and single or low dimensional output mappings, Recurrent Neural Networks for the prediction and extrapolation of processes and the Proper Orthogonal Decomposition method for the reduction of high dimensional data and, in combination with Radial Basis Functions, for the nonlinear interpolation of high dimensional output data. In [26], an artificial neural network has been employed as a surrogate model for deterministic real time analyses of mechanised tunnelling. In this paper, the benefits of Artificial Neural Networks and Proper Orthogonal Decomposition approaches are combined within a hybrid RNN-GPOD surrogate model for Type 1 Mapping of processes with a low dimensional input and a high dimensional output. 


\subsection{Hybrid surrogate modelling scheme}

For the support of decisions during the tunnel advancement process, predictions of expected surface settlements at multiple surface locations are required. For predefined (critical) sections of the tunnelling project, the analysis model needs to describe the dependencies between time variant deterministic process parameters $\mathbf{P}(t)$ and interval parameters $\overline{\mathbf{X}}$ related to the geotechnical model and the corresponding uncertain system response, such as time variant interval surface settlements $\overline{\mathbf{S}}(t)$. This motivates to generate a surrogate model with multiple interval output process prediction capabilities considering interval input data, i.e. Type 1 Mapping according to Section 3.1. Therefore, an extension of the hybrid surrogate model introduced in [27] is proposed to predict interval settlement fields, see Figure 5.

Figure 5a) shows a block diagram of the necessary computation in the offline stage, i.e. in the design phase of a tunnelling project, to set up the interval input-output data surrogate model. Firstly, a surrogate model is generated for a tunnel drive through a specific tunnel section with deterministic inputs (geotechnical parameters $\mathbf{X}$, time variant steering parameters $\mathbf{P}(t)$ ) and deterministic outputs (time variant surface settlements $\mathbf{S}(t)$ ) By varying the input parameters (design of experiments) of the numerical model ekate used to simulate the mechanised tunnelling process from time step 1 to $N$, a set of deterministic input-output data is collected. The next step is to compute the same system outputs for uncertain geotechnical parameters $\overline{\mathbf{X}}$ in the context of an interval analysis. This is also accomplished in the offline stage using the surrogate model with a deterministic input-output mapping together with an optimisation approach, e.g. the Particle Swarm Optimisation approach, developed in [27]. The obtained results are patterns of interval and deterministic input parameters and the corresponding interval surface settlement field from the time steps 1 to $N$. These patterns are used to create a hybrid RNN-GPOD surrogate model for interval data based on the midpoint-radius representation, which is described in the next subsections.

Figure $5 \mathrm{~b}$ ) presents how to apply the proposed surrogate model in the online stage, i.e. during the tunnel construction. The idea is that the surrogate model is operated, adopting the actually recorded steering parameters from the previous time steps 1 to $n$, to predict the interval bounds of the expected surface settlement field of the next time step $n+1$ with assumed (chosen) values of the steering parameters and given ranges of the geotechnical parameters. For this purpose, the surrogate model proposed in [27] is extended to allow processing of interval data. In a first step, trained Recurrent Neural Networks are employed for the midpoints and the radiuses to predict the interval bounds of settlements at several monitoring points for the next time step $n+1$. In a second step, the complete time variant interval surface settlement field from time step 1 to $n$ is approximated by trained Proper Orthogonal Decomposition Radial Basis Functions surrogate models for the midpoints and the radiuses. Finally, the Gappy Proper Orthogonal Decomposition and the Non-Negative Matrix Factorisation (NNMF) approaches are adopted to reproduce and predict the complete interval settlement field in the time step $n+1$ based on a combination of the results from the two previous methods. The predicted results are then included into the available inter- 


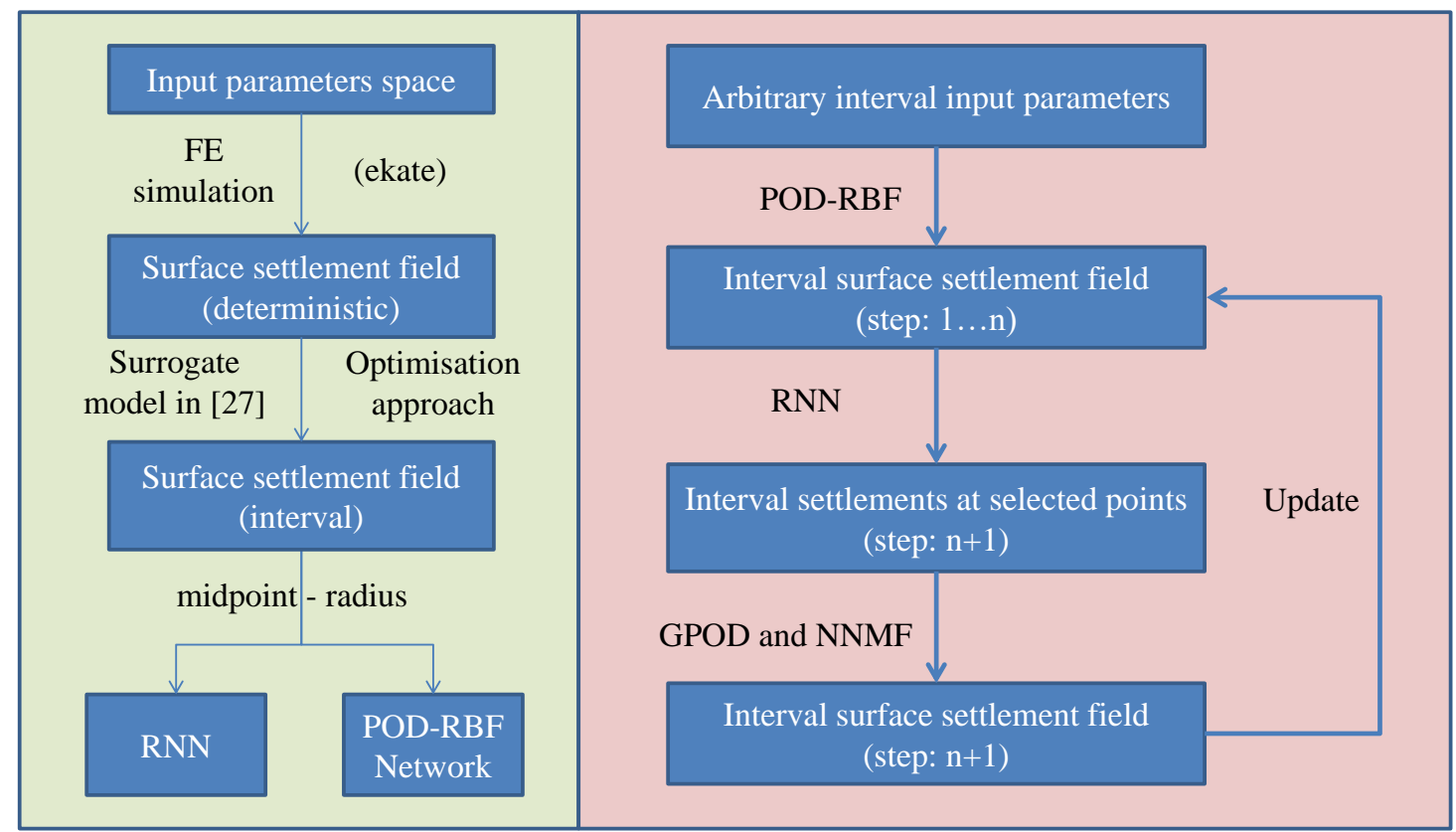

Figure 5: Scheme of the proposed hybrid surrogate modelling approach with interval data. a) Offline stage; b) Online stage.

val data set and the procedure is repeated for the subsequent time steps. The algorithm of the proposed extended surrogate modelling approach is summarised in Table 2.

\subsection{Process prediction with recurrent neural networks for interval data}

Artificial neural networks are widely used as surrogate models in structural mechanics, see e.g. [19, 20]. Often, multilayer perceptrons with a feed forward architecture, see [40], are utilized to learn functional relationships in data. In [41] and [42], feed forward neural networks are used to predict surface settlements resulting from machine driven tunnelling. The networks are trained with data obtained from numerical simulations using the process-oriented finite element model for mechanised tunnelling described in Section 2.

For the approximation and prediction of dependencies between structural processes, recurrent neural networks (RNNs) are beneficial, see e.g. [36]. RNNs are able to learn dependencies between data series without considering time as additional input parameter. This enables to capture time-dependent phenomena in data series and predict 


\section{Offline stage}

1: Generate a finite element model representing the tunnel advancement process within predefined sections of the tunnel project from time step $n=1, \ldots, N$ using the process oriented computational model ekate

2: Define deterministic input parameters (geotechnical parameters $\mathbf{X}$ and steering parameters ${ }^{[n]} \mathbf{P}$ ) with typical ranges and scenarios for possible variations of the steering parameters.

3: Run numerical simulations with different patterns of deterministic input parameters (design of experiment)

4: Store the numerical results of the deterministic settlement fields in the vector ${ }^{[n]} \mathbf{S}$

5: Define intervals of the geotechnical parameters $\overline{\mathrm{X}}$

6: Run the deterministic surrogate model in [27] for different patterns of $\overline{\mathrm{X}}$ and scenarios of deterministic steering parameters ${ }^{[n]} \mathbf{P}$ within an interval analysis optimisation approach

7: Store the lower and upper bounds ${ }^{[n]} \overline{\mathbf{S}}$ of the interval settlement field

8: Provide interval settlement data at selected monitoring points ${ }^{[n]} \bar{s}_{i_{R N N}}$ to train and test the RNN

9: Provide interval settlement data for the complete settlement field ${ }^{[n]} \overline{\mathbf{S}}$ to train the POD-RBF

\section{Online stage}

1: Input: Interval of the geotechnical parameters $\overline{\mathbf{X}}$, the recorded history of steering parameters ${ }^{[1]} \mathbf{P}$ to ${ }^{[n]} \mathbf{P}$ for time steps 1 to $n$ and assumed values of ${ }^{[n+1]} \mathbf{P}$ for time step $n+1$

2: Approximate the lower and upper bounds of the complete interval settlement field from time step 1 to $n$ (by POD-RBF)

3: Predict the lower and upper bounds of the interval settlements at the selected monitoring points ${ }^{[n+1]} \bar{s}_{i_{R N N}}$ for the next time step $n+1$ (by RNN)

4: Predict the interval bounds of the complete settlement field ${ }^{[n+1]} \overline{\mathbf{S}}$ of next time step $n+1$ (by GPOD and NNMF)

5: Update the interval bounds of the complete settlement field from time step 1 to $n+1$

6: Repeat steps 3 and 4 with updated inputs

Table 2: Algorithm of the hybrid RNN-GPOD surrogate modelling approach to predict interval bounds of the expected surface settlement field in time step $n+1$.

(extrapolate) further structural responses. The layered network structure of the RNNs is similar to the architecture of feed forward neural networks. But in addition to the neurons, so-called context neurons are used to consider the structural history, see Figure 6. The RNN architecture is based on the Elman [43] and Jordan [44] networks. However, in the present RNN formulation, multiple delayed context layers are considered, see [20]. For each hidden and each output neuron, a context neuron is assigned. These context neurons send multiple time delayed context signals to the hidden and output neurons. 


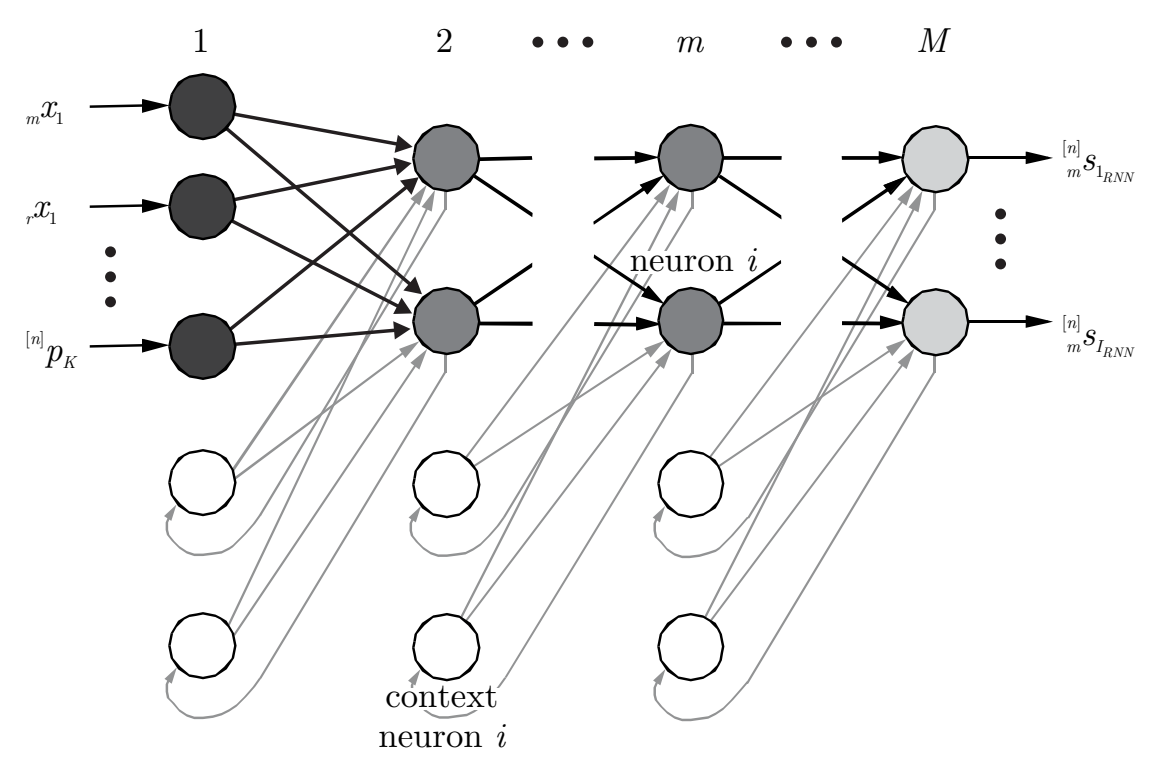

Figure 6: Recurrent neural network (midpoint RNN) for interval settlement process predictions.

In order to process interval data with RNNs, the midpoint-radius representation of interval data is suggested in this paper. For the prediction of the time variant interval settlement at selected monitoring points, two separate RNNs for the midpoints and the radiuses, denoted as midpoint RNN and radius RNN, are generated. In each time step $n$, the input signals of both networks (i.e. the midpoints ${ }_{m} x_{j}$ and radiuses ${ }_{r} x_{j}$ of the time constant geotechnical interval parameters $\bar{x}_{j}$ and the deterministic steering parameters ${ }^{[n]} p_{k}$ ) are processed layer by layer to get the network outputs (i.e. the midpoints ${ }_{m}^{[n]} s_{i_{R N N}}$ or radiuses ${ }_{r}^{[n]} s_{i_{R N N}}$ of the interval settlements ${ }^{[n]} \bar{s}_{i_{R N N}}$ at the selected monitoring points $i_{R N N}$ ), see Figure 6 exemplified for the midpoint RNN.

The signals in the hidden and output neurons of the corresponding midpoint RNN and radius $\mathrm{RNN}$ are computed by

$$
{ }^{[n]} x_{i}^{(m)}=\varphi_{i}^{(m)}\left(\sum_{h=1}^{H}\left[{ }^{[n]} x_{h}^{(m-1)} \cdot w_{i h}^{(m)}\right]+\sum_{q=1}^{Q}\left[\sum_{d=1}^{D}\left[{ }^{[n-d]} x_{q}^{(m)} \cdot{ }^{d} c_{i q}^{(m)}\right]\right]+b_{i}^{(m)}\right) .
$$

In Eq. (9), $\varphi_{i}^{(m)}($.$) is the activation function of neuron i$ in layer $(m)$. The argument $\nu_{i}^{(m)}$ of the activation function is computed as a sum of all output signals $x_{h}^{(m-1)}$ of the previous layer $(m-1)$, multiplied by their synaptic weights $w_{i h}^{(m)}$ plus a sum of all $[n-1], \ldots,[n-D]$ delayed prior output signals ${ }^{[n-d]} x_{q}^{(m)}$ of the hidden neurons in the same layer $(m)$ multiplied by their context weights ${ }^{d} c_{i q}^{(m)}$ and an additional bias value $b_{i}^{(m)}$.

Different types of activation functions $\varphi_{i}^{(m)}($.$) can be used to process the signals$ in the neurons. Because of the used gradient based training algorithm, they must be 
differentiable. Here, the hyperbolic tangent function

$$
\varphi(\nu)=\tanh (\nu)=\frac{\exp (\nu)-\exp (-\nu)}{\exp (\nu)+\exp (-\nu)}
$$

is used in the hidden neurons and the linear activation function

$$
\varphi(\nu)=\nu
$$

in the output neurons of the midpoint RNN. For the radius RNN, the logistic sigmoid function

$$
\varphi(\nu)=\frac{1}{1+\exp (-\nu)}
$$

is used in the hidden neurons and the positive linear function

$$
\varphi(\nu)= \begin{cases}\nu, & \text { if } \nu \geq 0 \\ 0, & \text { if } \nu<0\end{cases}
$$

is used in the output neurons to fulfil the constraint of positive outputs for the radiuses.

The synaptic weights $w_{i h}^{(m)}$, the context weights ${ }^{d} c_{i q}^{(m)}$ for each $d=1, \ldots, D$ delayed time step and the bias values $b_{i}^{(m)}$ are unknown network parameters, which are identified within the network training. The RNNs are trained by the LevenbergMarquardt backpropagation algorithm.

\subsection{Interpolation with Proper Orthogonal Decomposition for in- terval data}

The Proper Orthogonal Decomposition (POD) technique has gained wide popularity in data analysis, since it is capable of deriving a reduced order set of basis functions, which can represent the original high-dimensional data with a high level of accuracy. A brief overview of the POD method is presented in [45], [46]. The POD approach is applied in various fields under different names such as Principal Component Analysis [47] in statistics, Singular Value Decomposition [48] in linear algebra and KarhunenLoeve Decomposition [49], [50] in signal processing. Nowadays, the method is applied extensively in computational mechanics, e.g. in fluid dynamics [51], [52], aerodynamics [53] and nonlinear solid mechanics [54], [55]. In combination with Radial Basis Functions (RBF), the POD is used as surrogate model to solve inverse problems, see e.g. [56, 57, 24, 58].

In the paper, two separate POD-RBF surrogate models for the midpoints and the radiuses are created to produce quick predictions of the system response (i.e. the interval settlement field) corresponding to an arbitrary set of input parameters (realisations of geotechnical interval parameters and deterministic steering parameters) by interpolation between the sample data points. Before the midpoint-radius representation for POD-RBF with interval data is developed, a short review on the deterministic POD-RBF is presented next. 
The first step in creating a POD basis is to obtain a collection of $M$ snapshots of possible system solutions (settlements) by varying the input parameters, where each snapshot consists of $N$ output values (all settlements ${ }^{[n]} s_{i}$ of time steps $1, \ldots, n$ at all points $i=1, \ldots, I$ of the settlement field) corresponding to a specific set (scenario) of input parameters (one realisation with geotechnical parameters $x_{j}$ and steering parameters ${ }^{[n]} p_{k}$ for time steps $1, \ldots, n$ ). The obtained data are structured in the format of a so-called snapshot matrix $\mathbf{S}$ which possesses $N=n \cdot I$ rows and $M$ columns, where $M$ corresponds to the number of scenarios of input parameters. The POD basis vectors can be obtained by a Single Value Decomposition of the matrix $\mathbf{S}$ or by solving the eigenvalue problem of the sample covariance matrix $\mathbf{C}=\mathbf{S}^{\mathrm{T}} \cdot \mathbf{S}$. Employing the latter approach in this paper, the POD basis is expressed as a linear transformation of the snapshot matrix $\mathbf{S}$

$$
\Phi=\mathbf{S} \cdot \mathbf{V},
$$

where $\mathbf{V}$ is a matrix obtained by solving the following eigenvalue problem

$$
\mathbf{C} \cdot \mathbf{V}=\boldsymbol{\Lambda} \cdot \mathbf{V}
$$

The matrix $\mathbf{V}$ contains eigenvectors corresponding to eigenvalues of the matrix $\mathbf{C}$. Each eigenvalue $\lambda_{i}$ contained in the matrix $\lambda$ represents the variance of the data set in the direction of the corresponding POD mode. The eigenvalue $\lambda_{i}$ can be shown to be a measure of the approximation capabilities of the $i^{t h}$ basis function. Typically, depending on the desired accuracy, only $K \ll M$ basis functions are kept for the approximation of the snapshot matrix $\mathbf{S}$. The resulting matrix consisting of the first $K$ POD modes is denoted as truncated POD basis matrix $\hat{\boldsymbol{\Phi}}$.

The exact and approximated snapshot matrix $\mathbf{S}$ is represented by

$$
\begin{aligned}
& \mathbf{S}=\boldsymbol{\Phi} \cdot \mathbf{A}, \\
& \mathbf{S} \approx \hat{\boldsymbol{\Phi}} \cdot \hat{\mathbf{A}},
\end{aligned}
$$

respectively. The matrices $\mathbf{A}$ and $\hat{\mathbf{A}}$ are the amplitude matrix and the truncated amplitude matrix, respectively. From Eqs. (16) and (17), A and $\hat{\mathbf{A}}$ can be constructed as

$$
\begin{aligned}
& \mathbf{A}=\boldsymbol{\Phi}^{\mathrm{T}} \cdot \mathbf{S}, \\
& \hat{\mathbf{A}} \approx \hat{\boldsymbol{\Phi}}^{\mathrm{T}} \cdot \mathbf{S} .
\end{aligned}
$$

It should be noted, that at this step $\hat{\mathbf{A}}$ contains only the information of the scenarios of input parameters used to create the snapshot matrix.

To approximate the system behaviour related to intermediate values of input parameters that are not included in the snapshot data, an interpolation is performed to determine the truncated amplitude matrix $\hat{\mathbf{A}}$, assuming that $\hat{\mathbf{A}}$ is a smooth function of input parameters. More specifically, each amplitude vector is defined as a linear combination of a set of vectors $\mathbf{F}^{i}=\left[f_{1}\left(\mathbf{Q}^{i}\right) \ldots f_{j}\left(\mathbf{Q}^{i}\right) \ldots f_{M}\left(\mathbf{Q}^{i}\right)\right]^{T}$, with $f_{j}\left(\mathbf{Q}^{i}\right)$ as predefined interpolation functions of the input parameter scenarios $\mathbf{Q}^{i}$ and an unknown coefficient matrix $\mathbf{B}$ as

$$
\hat{\mathbf{A}}^{i}=\mathbf{B} \cdot \mathbf{F}^{i} .
$$


The choice of $f_{j}\left(\mathbf{Q}^{i}\right)$ can be arbitrary. A suitable option is to employ Radial Basis Functions (RBFs) as interpolation functions due to their good approximation and smoothing properties. In this paper, the inverse multiquadric radial basis function, see $[59,60]$, is used as interpolation function. Each element of the vector $\mathbf{F}^{i}$ is computed according to

$$
f_{j}\left(\mathbf{Q}^{i}\right)=f_{j}\left(\left|\mathbf{Q}^{i}-\mathbf{Q}^{j}\right|\right)=\frac{1}{\sqrt{\left|\mathbf{Q}^{i}-\mathbf{Q}^{j}\right|^{2}+c^{2}}},
$$

with $c$ as a smoothing factor selected as

$$
c=0.815\left(\frac{1}{M} \sum_{i=1}^{M} d^{i}\right),
$$

and $d^{i}$ is the distance between the $i$ th data point and its neighbour [61]. The matrix $\mathbf{F}$ containing the interpolation functions can be constructed from all vectors $\mathbf{F}^{i}$ of input parameters that are used to generate the snapshots. Using $\mathbf{F}$, the truncated amplitude matrix $\hat{\mathbf{A}}$ is given by

$$
\hat{\mathbf{A}}=\mathbf{B} \cdot \mathbf{F},
$$

From Eqs. (23) and (19), the coefficient matrix B is determined. Finally, an approximation of the output system response (settlement field) corresponding to an arbitrary set of input parameters (geotechnical and steering parameters) is obtained by

$$
\mathbf{S}^{a} \approx \hat{\mathbf{\Phi}} \cdot \mathbf{B} \cdot \mathbf{F}^{a} .
$$

The algorithm for POD-RBF procedure for deterministic data is summarised in Table 3 .

For the processing of interval data by means of the midpoint-radius representation (see Section 3) the POD-RBF algorithm is applied separately for the midpoints and radiuses. A constraint for the radiuses is that the predicted results must be nonnegative. Since the approximation with POD-RBF is just an interpolation between sample points with non-negative outputs, the constraint is also satisfied for the approximated results. The summary of the POD-RBF algorithm with interval data is presented in Table 4.

\subsection{Predicting missing interval data}

For predicting missing interval data within the hybrid surrogate modelling strategy, the midpoints and radiuses of the full settlement field at time step $n+1$ are predicted separately using the settlement fields of all prior time steps and the RNN predictions at the monitoring points ${ }^{[n+1]} \bar{s}_{i_{R N N}}$, see Section 4.1. The midpoints are predicted with the Gappy Proper Orthogonal Decomposition (GPOD) approach (Subsection 4.4.1). However, this approach cannot be used for predicting the radiuses of the interval data, since it does not guarantee to fullfil the non-negativity constraint. Therefore, the so-called Non-Negative Matrix Factorisation is utilized for the radiuses (Subsection 4.4.2). 
Input: Deterministic snapshots output matrix $\mathbf{S}$, snapshots input matrix $\mathbf{Q}$, arbitrary input vector $\mathbf{Q}^{a}$, desired accuracy $E$

Output: System response vector $\mathbf{S}^{a}$

A - Compute the truncated POD basis matrices $\hat{\boldsymbol{\Phi}}$

1: Compute covariance matrices $\mathbf{C}=\mathbf{S}^{\mathrm{T}} \cdot \mathbf{S}$

2: Compute eigenvalue decomposition $[\boldsymbol{\Psi}, \boldsymbol{\Lambda}]=\operatorname{eig}(\mathbf{C})$

3: Set $\lambda_{i}=\boldsymbol{\Lambda}_{i i}$, for $i=1, \ldots, M$

4: Set $\boldsymbol{\Phi}_{i}=\mathbf{S} \cdot \boldsymbol{\Psi}_{-, i} / \sqrt{\lambda_{i}}$, where $\boldsymbol{\Psi}_{-, i}$ is the $i$ th column of matrix $\boldsymbol{\Psi}$

5: Define $K$ based on the desired accuracy $E$ with $\sum_{i=1}^{K} \lambda_{i} / \sum_{i=1}^{M} \lambda_{i} \geq E$

6: Obtain truncated POD basis $\hat{\Phi}$ by taking the first $K$ columns of $\Phi$

B - Interpolation

1: Compute $f_{j}\left(\mathbf{Q}^{i}\right)$, with $i, j=1, \ldots, M$

2: Form matrix $\mathbf{F}$ from previous step

3: Compute $\hat{\mathbf{A}}=\hat{\boldsymbol{\Phi}}^{\mathrm{T}} \cdot \mathbf{S}$

4: Compute $\mathbf{B}$ based on $\mathbf{F}, \hat{\mathbf{A}}$

5: Compute $f_{j}\left(\mathbf{Q}^{a}\right)$, with $j=1, \ldots, M$

6: Form vector $\mathbf{F}^{a}$ from previous step

7: Return the system response vector $\mathbf{S}^{a}=\hat{\boldsymbol{\Phi}} \cdot \mathbf{B} \cdot \mathbf{F}^{a}$

Table 3: POD-RBF algorithm to predict deterministic system response from an arbitrary set of input parameters.

Input: Interval snapshots output matrix $\mathbf{S}$, interval snapshots input matrix $\mathbf{Q}$, arbitrary interval input vector $\overline{\mathbf{Q}}^{a}$, desired accuracy $E$

Output: Interval system response vector $\overline{\mathbf{S}}^{a}$

1: Transform interval data into the type of midpoint and radius data according to Eqs. (2) and (3)

$$
\begin{aligned}
& { }_{m} \mathbf{S}=\left({ }_{l} \mathbf{S}+{ }_{u} \mathbf{S}\right) / 2 ; \quad{ }_{r} \mathbf{S}=\left({ }_{u} \mathbf{S}-{ }_{l} \mathbf{S}\right) / 2 \\
& { }_{m} \mathbf{Q}=\left({ }_{l} \mathbf{Q}+{ }_{u} \mathbf{Q}\right) / 2 ; \quad{ }_{r} \mathbf{Q}=\left({ }_{u} \mathbf{Q}-{ }_{l} \mathbf{Q}\right) / 2 \\
& { }_{m} \mathbf{Q}^{a}=\left({ }_{l} \mathbf{Q}^{a}+{ }_{u} \mathbf{Q}^{a}\right) / 2 ; \quad{ }_{r} \mathbf{Q}^{a}=\left({ }_{u} \mathbf{Q}^{a}-{ }_{l} \mathbf{Q}^{a}\right) / 2
\end{aligned}
$$

2: Form the deterministic input matrix and input vector including both midpoint and radius representations $\left.{ }_{d} \mathbf{Q}={ }_{[m} \mathbf{Q} ;{ }_{r} \mathbf{Q}\right]$ and ${ }_{d} \mathbf{Q}^{a}=\left[{ }_{m} \mathbf{Q}^{a} ;{ }_{r} \mathbf{Q}^{a}\right]$

3: Compute the system response corresponding to midpoint and radius representation ${ }_{m} \mathbf{S}^{a}$ and ${ }_{r} \mathbf{S}^{a}$

${ }_{m} \mathbf{S}^{a}=\mathrm{POD}_{-} \mathrm{RBF}\left({ }_{m} \mathbf{S},{ }_{d} \mathbf{Q},{ }_{d} \mathbf{Q}^{a}, E\right)$, see Table 3

$\left.{ }_{r} \mathbf{S}^{a}=\mathrm{POD}_{-} \operatorname{RBF}_{r} \mathbf{S},{ }_{d} \mathbf{Q},{ }_{d} \mathbf{Q}^{a}, E\right)$, see Table 3

4: Compute the upper and lower bounds of the system response vector according to Eqs. (4) and (5)

$$
\begin{aligned}
& { }_{u} \mathbf{S}^{a}={ }_{m} \mathbf{S}^{a}+{ }_{r} \mathbf{S}^{a} \\
& { }_{l} \mathbf{S}^{a}={ }_{m} \mathbf{S}^{a}-{ }_{r} \mathbf{S}^{a}
\end{aligned}
$$

5: Return the interval system response vector $\left.\overline{\mathbf{S}}^{a}={ }_{l} \mathbf{S}^{a} ;{ }_{u} \mathbf{S}^{a}\right]$

Table 4: POD-RBF algorithm to predict lower and upper bounds of interval system responses from an arbitrary set of interval and deterministic input parameters. 


\subsubsection{Gappy proper orthogonal decomposition for midpoint predictions}

Without any missing data, an arbitrary snapshot $\mathbf{S}^{j}$ which belongs to a set of snapshots can be approximated as a linear combination of the first $K$ POD basis vectors $\hat{\Phi}$ and an amplitude vector $\hat{\mathbf{A}}^{j}$ as described in the previous subsection. The amplitude vector is calculated by minimising the error norm

$$
\min .\left\|\mathbf{S}^{j}-\hat{\Phi} \cdot \hat{\mathbf{A}}^{j}\right\|_{L^{2}}^{2}
$$

The same least square approach can be effectively used to predict missing data of an incomplete data snapshot. However, due to missing elements, the $L^{2}$ norm in Eq. (25) cannot be evaluated correctly. As a remedy, the GPOD algorithm, introduced in [51], is using the concept of a gappy norm based on the available data. This method is based on the conventional POD method for solving an unconstrained optimisation problem, which approximately reconstructs the full data from the incomplete one. This concept is already successfully applied in aerodynamics [53] for the data recovery of the flow field.

Firstly, the GPOD algorithm is using a vector $\mathbf{m}$ to identify available and missing data. Each element of $\mathbf{m}$ is defined as

$$
\begin{array}{ll}
\mathbf{m}_{i}=0, & \text { for locations of unknown or missing data, } \\
\mathbf{m}_{i}=1, & \text { for locations of known data. }
\end{array}
$$

Given the POD basis ${ }_{m} \boldsymbol{\Phi}$ of an output system response snapshot set ${ }_{m} \mathbf{S}$, where all snapshots are known, and let ${ }_{m} \mathbf{S}^{*}$ be another solution vector, that has some elements missing, with a corresponding mask vector $\mathbf{m}$, the goal is to produce the complete vector from ${ }_{m} \mathbf{S}^{*}$ with the assumption, that ${ }_{m} \mathbf{S}^{*}$ can be characterised by the existing snapshots set ${ }_{m} \mathbf{S}$. An intermediate repaired vector ${ }_{m} \widetilde{\mathbf{S}}^{*}$ can be expressed as truncated POD basis vectors ${ }_{m} \hat{\boldsymbol{\Phi}}$

$$
{ }_{m} \widetilde{\mathbf{S}}^{*} \approx{ }_{m} \hat{\mathbf{\Phi}} \cdot{ }_{m} \hat{\mathbf{A}}^{*}
$$

By minimizing the error $E=\left\|{ }_{m} \mathbf{S}^{*}-{ }_{m} \widetilde{\mathbf{S}}^{*}\right\|_{n}^{2}$, the coefficient vector ${ }_{m} \hat{\mathbf{A}}^{*}$ can be computed by solving the least squares or linear regression problem

$$
{ }_{m} \mathbf{M} \cdot{ }_{m} \hat{\mathbf{A}}^{*}={ }_{m} \mathbf{R}
$$

with

$$
{ }_{m} \mathbf{M}=\left({ }_{m} \hat{\boldsymbol{\Phi}}^{\mathrm{T}},{ }_{m} \hat{\boldsymbol{\Phi}}\right), \quad{ }_{m} \mathbf{R}=\left({ }_{m} \hat{\boldsymbol{\Phi}}^{\mathrm{T}},{ }_{m} \mathbf{S}^{*}\right) .
$$

Solving Eq. (27) with ${ }_{m} \hat{\mathbf{A}}^{*}$ computed from Eq. (28), the intermediate repaired vector ${ }_{m} \widetilde{\mathbf{S}}^{*}$ is obtained. Finally, by replacing the missing elements in ${ }_{m} \mathbf{S}^{*}$ by the respective components in ${ }_{m} \widetilde{\mathbf{S}}^{*}$ the complete vector of the output system response is predicted. 


\subsubsection{Non-negative matrix factorisation for radius predictions}

The GPOD approach cannot account for constraints with respect to the sign of the elements in the factorised matrices. Therefore, we propose using the Non-negative Matrix Factorisation (NNMF) as a matrix decomposition approach, which decomposes a non-negative matrix into two low-rank non-negative matrices for radius predictions. The NNMF was originally suggested by Paatero and Tapper [62,63] as a concept of Positive Matrix Factorisation concentrating on a specific application concerned with Byzantine algorithms. Due to its simple and effective algorithmic implementation and the potential value of part-based representation (see Lee and Seung [64]), the range of applications of the NNMF method has increased and includes now a large variety of scientific disciplines such as machine learning [65], signal processing [66], bioinformatics [67] and economics [68]. Modern NNMF algorithms can be divided into four categories: Standard, Constrained, Structured and Generalised NNMF. For a comprehensive review of the theory and the state-of-the-art of NNMF concepts we refer to [69]. In this paper, the Standard NNMF is utilized for radius predictions. This procedure is explained next.

Given a $(n \times m)$ non-negative matrix ${ }_{r} \mathbf{S}$ and a reduced rank $k$, the non-negative matrix factorisation algorithm is searching for two non-negative matrices $\mathbf{W}_{n \times k}$ and $\mathbf{H}_{k \times m}$ that approximate ${ }_{r} \mathbf{S}$ in the format

$$
{ }_{r} \mathbf{S} \approx \mathbf{W} \cdot \mathbf{H} .
$$

A corresponding optimisation problem is formulated in the Euclidean space as

$$
\min _{W, H} \frac{1}{2}\left\|{ }_{r} \mathbf{S}-\mathbf{W} \cdot \mathbf{H}\right\|_{F}^{2} \text { subject to, } \mathbf{W}, \mathbf{H} \geq 0 .
$$

Similar to the POD approach, $\mathbf{W}$ and $\mathbf{H}$ are denoted as the basis matrix and coefficient matrix, respectively. The minimisation problem in Eq. (31) can be solved by multiplicative update rules as introduced in [70]. This algorithm, which is easy to implement, does not guarantee to converge to a stationary point, see [71]. Therefore, the alternating non-negative least squares algorithm proposed in [71] is used in this paper, which ensures to converge.

The GPOD procedure can now be followed with some minor modifications to predict missing data of a non-negative vector containing the radiuses $\mathbf{S}^{+}$. The corresponding objective function $E=\left\|\mathbf{S}^{+}-\mathbf{W} \cdot \mathbf{H}\right\|_{n}^{2}$ to be minimised contains the distances between the available incomplete data vector and the predicted vector. The non-negative basis matrix $\mathbf{W}$ is assumed to be known from the available non-negative data matrix ${ }_{r} \mathbf{S}$. The coefficient vector $\mathbf{H}$ is obtained accounting for the non-negativity constraint by solving the non-negative least squares problem

$$
{ }_{r} \mathbf{M} \cdot \mathbf{H}={ }_{r} \mathbf{R},
$$

with

$$
{ }_{r} \mathbf{M}=\left(\mathbf{W}^{\mathrm{T}}, \mathbf{W}\right) \quad{ }_{r} \mathbf{R}=\left(\mathbf{W}^{\mathrm{T}},{ }_{r} \mathbf{S}^{+}\right) .
$$


In this paper, the non-negative-constrained least squares problem in Eq. (32) is solved by an algorithm introduced in [72]. Finally, similar to the GPOD approach, the goal to predict a complete vector for the radiuses of the output system response (the interval settlement field of time step $n+1$ ) is accomplished by replacing the missing elements in $\mathbf{S}^{+}$by the respective components in $\widetilde{\mathbf{S}}^{+}$, with $\widetilde{\mathbf{S}}^{+} \approx \mathbf{W} \cdot \mathbf{H}$.

\section{Numerical applications}

\subsection{Verification example}

The proposed hybrid surrogate modelling approach for interval data is illustrated by means of a verification example adopted from structural analysis. In this verification

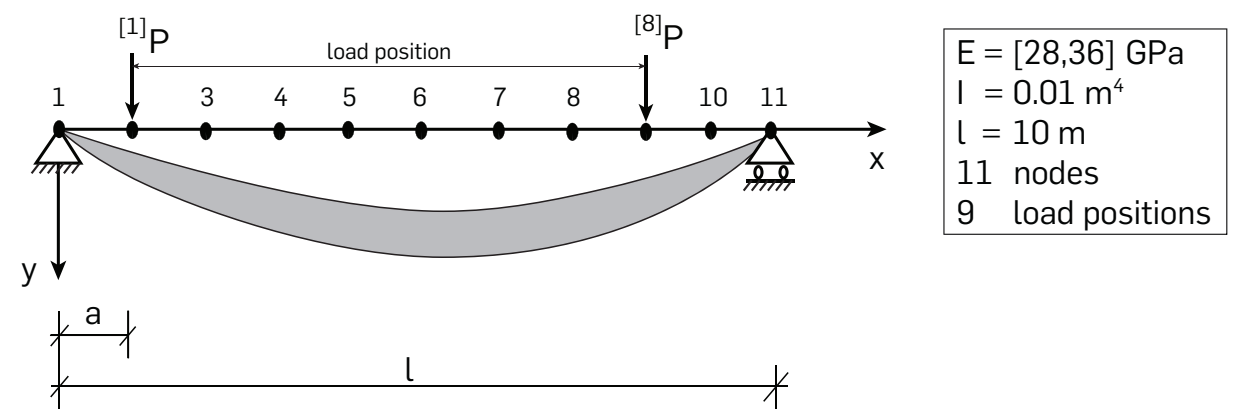

Figure 7: Verification example for the proposed hybrid surrogate model with interval data: Beam subjected to a moving single load.

example, the interval bounds of the deflection $\overline{\mathbf{S}}$ of a simply supported beam with a deterministic moving load $P$ along the length of the beam are investigated (see Figure 7). The modulus of elasticity of the beam is defined as an interval $\bar{E}=[28,36] \mathrm{GPa}$. The single load $P(t)$ is assumed as a time variant parameter moving along the beam from node 1 to 9 . The scenarios for the temporal change of $P(t)$ in each time step can be arbitrary, but $P(t)$ is assumed not to exceed a range from $10 \mathrm{kN}$ to $100 \mathrm{kN}$.

The objective of this verification example is to predict the interval deflection of the complete beam when the load $P(t)$ moves to the final position, i.e at $a=9 \mathrm{~m}$ with a given scenario for the moving load changing $P(t)$ stepwise from $a=1,2, \ldots 8 \mathrm{~m}$ as shown in Figure 8. It is assumed that only the interval deflections at nodes 2, 6 and 10 (i.e. for $x=1,5$ and $9 \mathrm{~m}$ ) can be measured. The deflections at the other nodes (the beam is discretised into 11 nodes) need to be predicted by the hybrid RNN-GPOD surrogate model. The final predictions of the surrogate model are compared with the analytical solution obtained from the following equations

$$
\begin{aligned}
& \bar{y}(x)=\frac{P(l-a) x}{6 l \bar{E} I}\left(l^{2}-x^{2}-(l-a)^{2}\right), \text { for } 0<x<a \\
& \bar{y}(x)=\frac{P(l-a)}{6 l \bar{E} I}\left[\frac{l}{l-a}(x-a)^{3}+\left(l^{2}-(l-a)^{2}\right) x-x^{3}\right], \text { for } a<x<l
\end{aligned}
$$




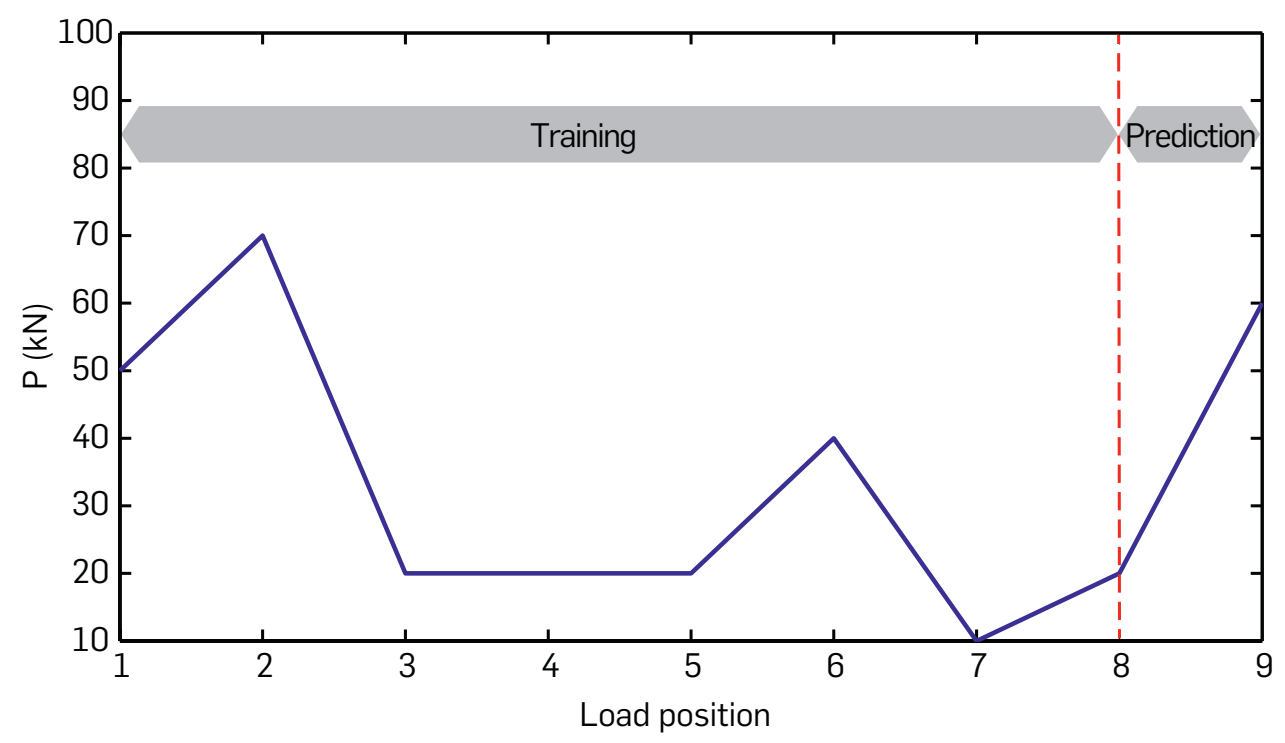

Figure 8: An arbitrary scenario of changing and moving load $P(t)$.

Theoretically, a deterministic surrogate model based on analytical results should be built to provide interval bounds for all beam nodes from step 1 to 8 using an optimisation approach. However, from the above equations, the upper bounds and lower bounds of the interval deflections can be directly calculated with interval arithmetic given the lower and upper bounds of $\bar{E}$. 48 snapshots are generated based on a combination of 8 different intervals of $\bar{E}$ and 6 scenarios of changing $P(t)$ to create the surrogate models. Each snapshot contains the interval displacements of 11 nodes of the beam from time step 1 to 8 . The snapshots data set is divided into sets for midpoints and radiuses as introduced in the previous subsections. The verification procedure can be split into the following steps:

1. Given an arbitrary interval of $\bar{E}$ and an arbitrary scenario of changing $P(t)$ from step 1 to 8 (which are not used to generate the surrogate models), the midpoints ${ }_{m} \mathbf{S}$ and the radiuses ${ }_{r} \mathbf{S}$ of the interval deflections at all 11 nodes from step 1 to 8 are obtained by the POD-RBF approach. Consequently, the upper and lower 
bounds are computed according to Eqs. (4) and (5).

$$
{ }_{m} \mathbf{S}=\left[\begin{array}{llllllll}
0.000 & 0.000 & 0.000 & 0.000 & 0.000 & 0.000 & 0.000 & 0.000 \\
0.455 & 1.103 & 0.405 & 0.403 & 0.428 & 0.734 & 0.116 & 0.208 \\
0.786 & 2.018 & 0.761 & 0.768 & 0.821 & 1.416 & 0.224 & 0.403 \\
0.983 & 2.593 & 1.020 & 1.056 & 1.145 & 1.991 & 0.317 & 0.571 \\
1.062 & 2.837 & 1.145 & 1.228 & 1.364 & 2.406 & 0.386 & 0.700 \\
1.039 & 2.798 & 1.145 & 1.258 & 1.445 & 2.610 & 0.425 & 0.777 \\
0.932 & 2.522 & 1.041 & 1.160 & 1.364 & 2.548 & 0.425 & 0.788 \\
0.758 & 2.057 & 0.853 & 0.960 & 1.145 & 2.190 & 0.378 & 0.720 \\
0.534 & 1.450 & 0.604 & 0.682 & 0.821 & 1.592 & 0.282 & 0.560 \\
0.275 & 0.749 & 0.312 & 0.354 & 0.428 & 0.836 & 0.150 & 0.306 \\
0.000 & 0.000 & 0.000 & 0.000 & 0.000 & 0.000 & 0.000 & 0.000
\end{array}\right]
$$

$$
r \mathbf{S}=\left[\begin{array}{llllllll}
0.000 & 0.000 & 0.000 & 0.000 & 0.000 & 0.000 & 0.000 & 0.000 \\
0.042 & 0.101 & 0.040 & 0.040 & 0.042 & 0.071 & 0.015 & 0.021 \\
0.073 & 0.185 & 0.074 & 0.076 & 0.080 & 0.136 & 0.029 & 0.042 \\
0.092 & 0.238 & 0.100 & 0.105 & 0.112 & 0.191 & 0.040 & 0.059 \\
0.099 & 0.260 & 0.112 & 0.122 & 0.133 & 0.231 & 0.049 & 0.072 \\
0.097 & 0.256 & 0.112 & 0.125 & 0.141 & 0.251 & 0.054 & 0.080 \\
0.087 & 0.231 & 0.102 & 0.115 & 0.133 & 0.245 & 0.054 & 0.081 \\
0.071 & 0.189 & 0.083 & 0.095 & 0.112 & 0.210 & 0.048 & 0.074 \\
0.050 & 0.133 & 0.059 & 0.068 & 0.080 & 0.153 & 0.036 & 0.058 \\
0.026 & 0.069 & 0.031 & 0.035 & 0.042 & 0.080 & 0.019 & 0.032 \\
0.000 & 0.000 & 0.000 & 0.000 & 0.000 & 0.000 & 0.000 & 0.000
\end{array}\right]
$$

The individual errors between approximation and analytical results of upper and lower bounds defined as

$$
\text { error }=\sqrt{\frac{\sum_{i=1}^{N}\left(\mathbf{S}_{\text {approx }}^{i}-\mathbf{S}_{\text {analytical }}^{i}\right)^{2}}{\sum_{i=1}^{N}\left(\mathbf{S}_{\text {analytical }}^{i}\right)^{2}}} \times 100 \%
$$

are $3.4 \%$ and $5.4 \%$, respectively.

2. Perform the POD procedure for the midpoint matrix ${ }_{m} \mathbf{S}$. The input are the snapshots matrix ${ }_{m} \mathbf{S}(11 \times 8)$ and the desired accuracy $E=99.9$. The output are 
the truncated POD basis vectors ${ }_{m} \hat{\Phi}(11 \times 2)$.

$$
{ }_{m} \hat{\boldsymbol{\Phi}}=\left[\begin{array}{rr}
0.000 & 0.000 \\
-0.157 & 0.320 \\
-0.293 & 0.482 \\
-0.386 & 0.414 \\
-0.436 & 0.183 \\
-0.443 & -0.102 \\
-0.410 & -0.328 \\
-0.338 & -0.412 \\
-0.238 & -0.361 \\
-0.123 & -0.207 \\
0.000 & 0.000
\end{array}\right]
$$

3. Perform the NNMF procedure for the radius matrix ${ }_{r} \mathbf{S}$. The input are the snapshots matrix ${ }_{r} \mathbf{S}(11 \times 8)$ and the reduced rank number $k=3$. The output are the non-negative basis vectors $\mathbf{W}(11 \times 3)$.

$$
\mathbf{W}=\left[\begin{array}{ccc}
0.000 & 0.000 & 0.000 \\
0.159 & 0.413 & 0.111 \\
0.303 & 0.520 & 0.217 \\
0.409 & 0.319 & 0.315 \\
0.461 & 0.073 & 0.395 \\
0.454 & 0.000 & 0.446 \\
0.395 & 0.141 & 0.452 \\
0.303 & 0.361 & 0.404 \\
0.198 & 0.457 & 0.304 \\
0.097 & 0.304 & 0.163 \\
0.000 & 0.000 & 0.000
\end{array}\right]
$$

4. Predict the midpoints and the radiuses of the interval deflections at nodes 2,6 and 10 (i.e. $x=1,5$ and $9 \mathrm{~m}$ ) in time step 9 by the midpoint $\mathrm{RNN}$ and the radius RNN, respectively. First, the patterns of input-output data at the 3 selected nodes are used for training and setting up the RNN surrogate models. Afterwards, an arbitrary input scenario of moving load $\mathrm{P}(\mathrm{t})$, e.g. the scenario illustrated in Figure 8 , is considered as the input. The outputs are the deflections at the 3 selected nodes (midpoints and radiuses). For the midpoint prediction a RNN with one hidden layer containing 7 hidden neurons was sufficient. The radius RNN required two hidden layers with 10 neurons per layer. In both networks, a delay of one time step has been selected for the context neurons.

$$
{ }_{m} \overline{\mathbf{S}}_{R N N}=\left[\begin{array}{l}
0.372 \\
1.165 \\
0.477
\end{array}\right] \quad{ }_{m} \overline{\mathbf{S}}_{\text {analytical }}=\left[\begin{array}{l}
0.311 \\
1.175 \\
0.514
\end{array}\right]
$$




$$
{ }_{r} \overline{\mathbf{S}}_{R N N}=\left[\begin{array}{c}
0.035 \\
0.142 \\
0.055
\end{array}\right] \quad{ }_{r} \overline{\mathbf{S}}_{\text {analytical }}=\left[\begin{array}{c}
0.039 \\
0.147 \\
0.064
\end{array}\right]
$$

The errors computed by Eq. (36) for the predicted midpoints and radiuses of the selected points are $5.4 \%$ and $6.6 \%$, respectively.

5. Predict intervals of deflections of all 11 nodes of the beam in time step 9. The input are the snapshots matrices ${ }_{m} \mathbf{S}(11 \times 8){ }_{r} \mathbf{S}(11 \times 8)$, the mask vector $\mathbf{m}$, the incomplete vector ${ }_{m} \mathbf{S}^{*}$, and the incomplete vector ${ }_{r} \mathbf{S}^{*}$. The output is the complete vector $\overline{\mathbf{S}}^{*}$.

As introduced in the previous sections, the proposed surrogate model consists of three different parts: POD-RBF, RNN and GPOD-NNMF. To verify the capabilities of each component as well as of the combination of all three components, three variants of surrogate models are established and investigated in Table 5: The surrogate model A uses the results from the analytical solution instead of predicted results from the first two methods to show the capability of the GPOD-NNMF method. The surrogate model B replaces the predicted results from the second method with analytical results, i.e. it combines the analytical model with the POD-RBF and the GPOD-NNMF. Finally, the proposed surrogate model, characterised by the combination of the three methods is denoted as surrogate model $\mathrm{C}$.

Table 5: Investigation of different variants of surrogate models.

\begin{tabular}{ccc}
\hline Surrogate model & Upper bound error (\%) & Lower bound error (\%) \\
\hline A & 3.9 & 4.0 \\
B & 3.7 & 4.4 \\
C & 6.8 & 8.4 \\
\hline
\end{tabular}

$$
\overline{\mathbf{S}}_{\mathbf{A}}^{*}=\left[\begin{array}{l}
{[0.000,0.000]} \\
{[\mathbf{0 . 2 7 2}, \mathbf{0 . 3 5 0}]} \\
{[0.475,0.618]} \\
{[0.719,0.932]} \\
{[0.923,1.199]} \\
{[\mathbf{1 . 0 2 8}, \mathbf{1 . 3 2 1}]} \\
{[1.077,1.379]} \\
{[0.955,1.221]} \\
{[0.712,0.909]} \\
{[\mathbf{0 . 4 5 0}, \mathbf{0 . 5 7 9}]} \\
{[0.000,0.000]}
\end{array}\right] \quad \overline{\mathbf{S}}_{\mathbf{B}}^{*}=\left[\begin{array}{l}
{[0.000,0.000]} \\
{[\mathbf{0 . 2 7 2}, \mathbf{0 . 3 5 0}]} \\
{[0.468,0.625]} \\
{[0.720,0.932]} \\
{[0.937,1.193]} \\
{[\mathbf{1 . 0 2 8}, \mathbf{1 . 3 2 1}]} \\
{[1.073,1.381]} \\
{[0.942,1.231]} \\
{[0.695,0.923]} \\
{[\mathbf{0 . 4 5 0 , 0 . 5 7 9}]} \\
{[0.000,0.000]}
\end{array}\right]
$$




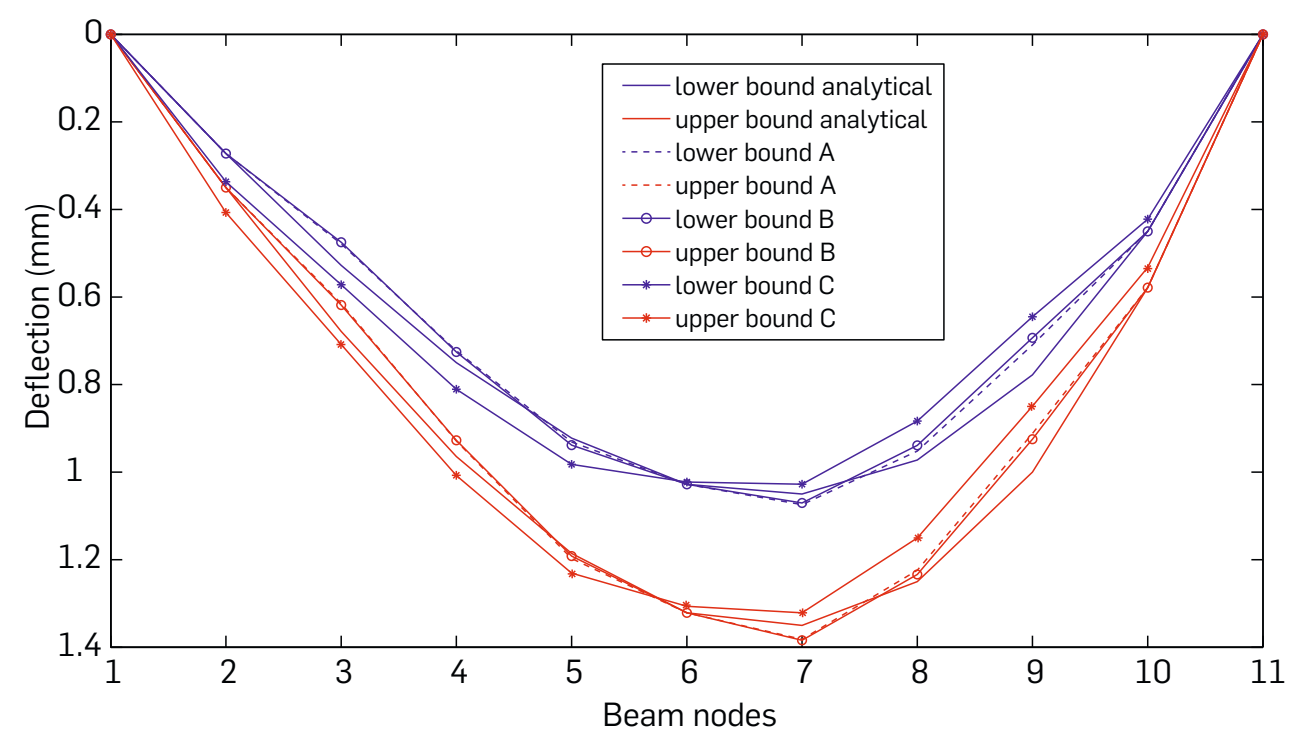

Figure 9: Comparison of predictions from different surrogate models (A, B and C) with the analytical solution.

$$
\overline{\mathbf{S}}_{\mathbf{C}}^{*}=\left[\begin{array}{l}
{[0.000,0.000]} \\
{[\mathbf{0 . 3 3 7}, \mathbf{0 . 4 0 7}]} \\
{[0.567,0.714]} \\
{[0.806,1.012]} \\
{[0.980,1.232]} \\
{[\mathbf{1 . 0 2 2}, \mathbf{1 . 3 0 7}]} \\
{[1.030,1.319]} \\
{[0.889,1.147]} \\
{[0.648,0.847]} \\
{[\mathbf{0 . 4 2 2}, \mathbf{0 . 5 3 2}]} \\
{[0.000,0.000]}
\end{array}\right] \quad \mathbf{S}_{\text {analytical }}^{*}=\left[\begin{array}{l}
{[0.000,0.000]} \\
{[\mathbf{0 . 2 7 2}, \mathbf{0 . 3 5 0}]} \\
{[0.528,0.679]} \\
{[0.750,0.963]} \\
{[0.922,1.186]} \\
{[\mathbf{1 . 0 2 8}, \mathbf{1 . 3 2 1}]} \\
{[1.050,1.350]} \\
{[0.972,1.250]} \\
{[0.778,1.000]} \\
{[\mathbf{0 . 4 5 0 , 0 . 5 7 9}]} \\
{[0.000,0.000]}
\end{array}\right]
$$

Figure 9 shows a comparison of predictions using different surrogate models together with the analytical solution. The errors for the predicted upper and lower interval bounds of all nodes using the complete proposed surrogate model are $6.8 \%$ and $8.4 \%$, respectively.

\subsection{Settlement predictions in mechanised tunnelling}

This section presents an application of the proposed surrogate modelling scheme for interval geotechnical data to a mechanised tunnelling process based on a synthetic example. The interval results obtained from the proposed method are compared with the reference solution based upon an optimisation approach presented in [27] in terms of prediction accuracy and computation time. The deterministic surrogate model is first trained by means of the finite element model ekate described in Section 2. Details of the capability of the surrogate model for processing deterministic data can 


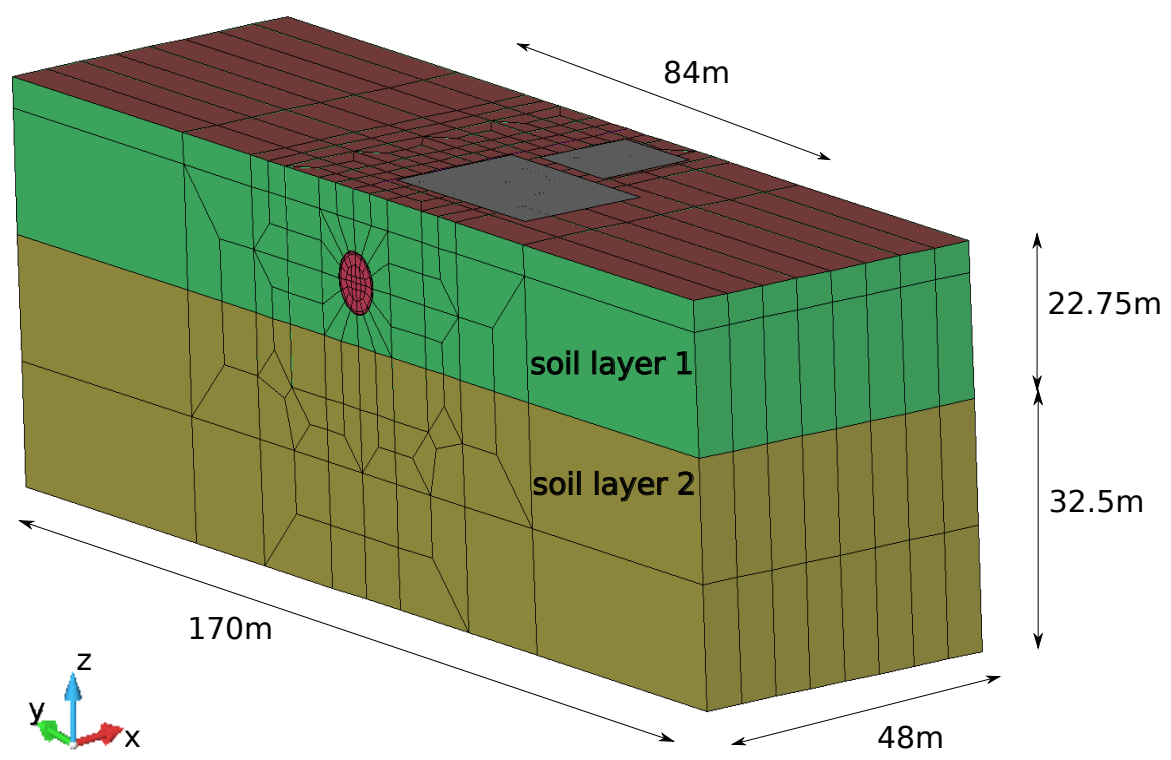

Figure 10: Numerical simulation model of a tunnel section. Geometry and finite element discretisation.

be found in $[73,27]$. Afterwards, the interval results computed with the optimisation approach are utilised to train the proposed hybrid RNN-GPOD surrogate model with interval data to predict the interval bounds of the surface settlements for further time steps of the mechanised tunnelling process.

In this example, a tunnel of $8.5 \mathrm{~m}$ diameter is assumed to be constructed by a tunnel boring machine with a centre line depth of $12.75 \mathrm{~m}$. The dimension of the soil block in this example are $48 \mathrm{~m}, 170 \mathrm{~m}$ and $55.25 \mathrm{~m}$ in X, Y, Z-direction, respectively. Figure 10 shows the geometry and the finite element discretisation of the tunnelling model. The effect of the stiffness of two existing buildings located at the surface is considered by placing rectangular plate-like substitute models with an equivalent thickness of $5 \mathrm{~m}$ and a stiffness of $50 \mathrm{GPa}$ at the top of the discretised soil body, which consists of 11,072 quadrilateral two-field finite elements with quadratic approximations for the displacements and linear approximations of the water pressure (Figure 10 and Figure 11).

The excavated domain comprises two layers of soft soil as shown in Figure 10. It is assumed, that the TBM advances completely within the clay layer (soil layer 1). In this application, the elastic modulus of layer 1 is taken as an uncertain parameter defined by an interval. A Drucker-Prager plasticity model is used to model the soil behaviour of both layers. The behaviour of the lining shell and the shield machine are assumed to be linear elastic. The tunnel advancement process is simulated via a step-by-step procedure including individual phases as described in Section 2.

Considering, that beyond a distance of $42 \mathrm{~m}$ in Y-direction from both sides of the 
tunnel axis, the surface settlements are almost zero, an effective rectangular surface area with 105 grid points as illustrated in Figure 11 is considered for the generation of the surrogate model. It is assumed, that the current state of the TBM advance cor-

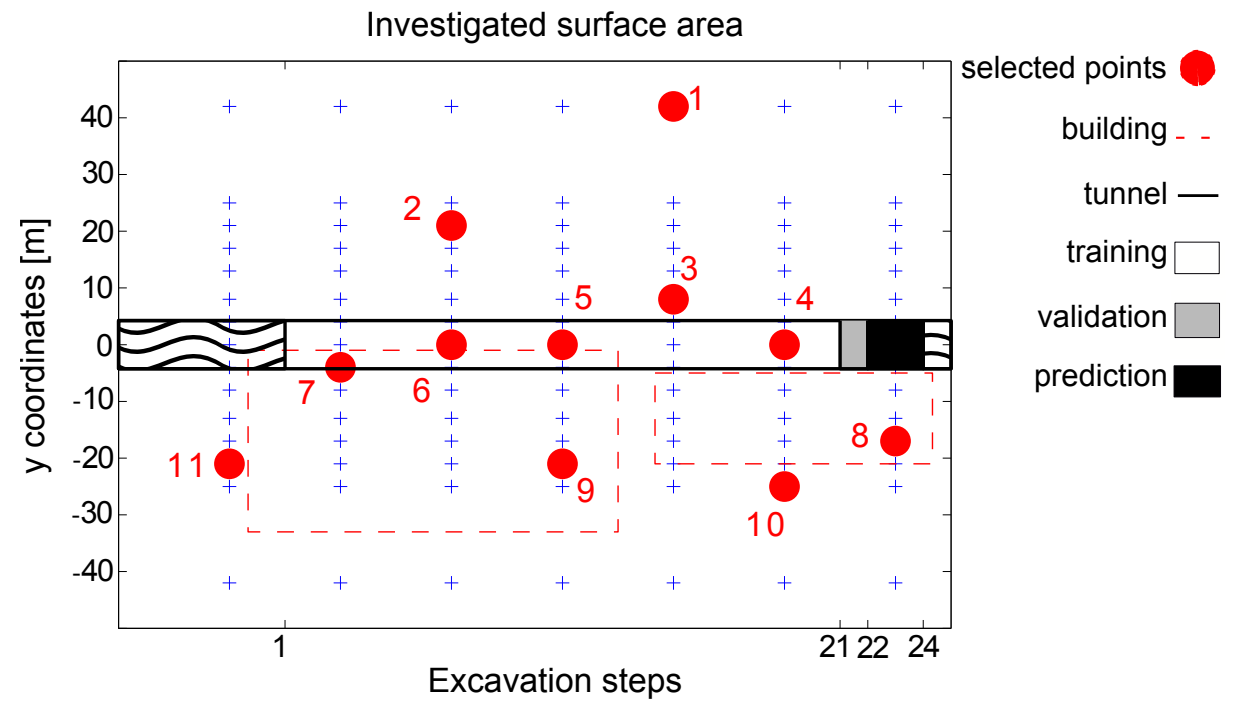

Figure 11: Surface area used for settlement predictions.

responds to the $22^{\text {nd }}$ step of the excavation process. The proposed hybrid surrogate modelling approach is employed to predict the complete interval surface displacement field in the subsequent excavation step (step 23) based upon the interval input parameter $\bar{E}_{1}$ of the soil layer 1 and the recorded history of the process parameters. In this application example, the interval settlements of 11 selected monitoring points are predicted by the RNN approach at time step $n+1$, i.e. for the advancement step 23 . Subsequently, the complete surface displacement field will be approximated with the GPOD and NNMF method under consideration of interval data.

In order to create the deterministic surrogate model, different parameters were varied within specified ranges and afterwards combined to generate simulation scenarios. In this study, these parameters are the Young modulus of the first soil layer $E_{1}$ and the grouting pressure at each excavation step ${ }^{[n]} P, n=1 \ldots 22$. The parameter range is 20 to $110 \mathrm{MPa}$ for $E_{1}$ and 130 to $230 \mathrm{kPa}$ for ${ }^{[n]} P$, respectively. As mentioned before, the proposed approach is compared with the reference solution obtained from employing an optimisation approach in an interval analysis assuming $\bar{E}_{1}$ as an interval $\bar{E}_{1}=[45,52] \mathrm{MPa}$, see [27]. For that purpose, the interval bounds of the surface field from time step 1 to 22 are constructed based on the deterministic surrogate model with the optimisation approach.

Figure 12 shows a comparison between the predicted settlements at all 105 points distributed within the area of influence of the tunnelling process and the reference solution obtained with the optimisation approach. The prediction shows a good accuracy with respect to the reference solution in both the upper and lower bounds. The errors, 


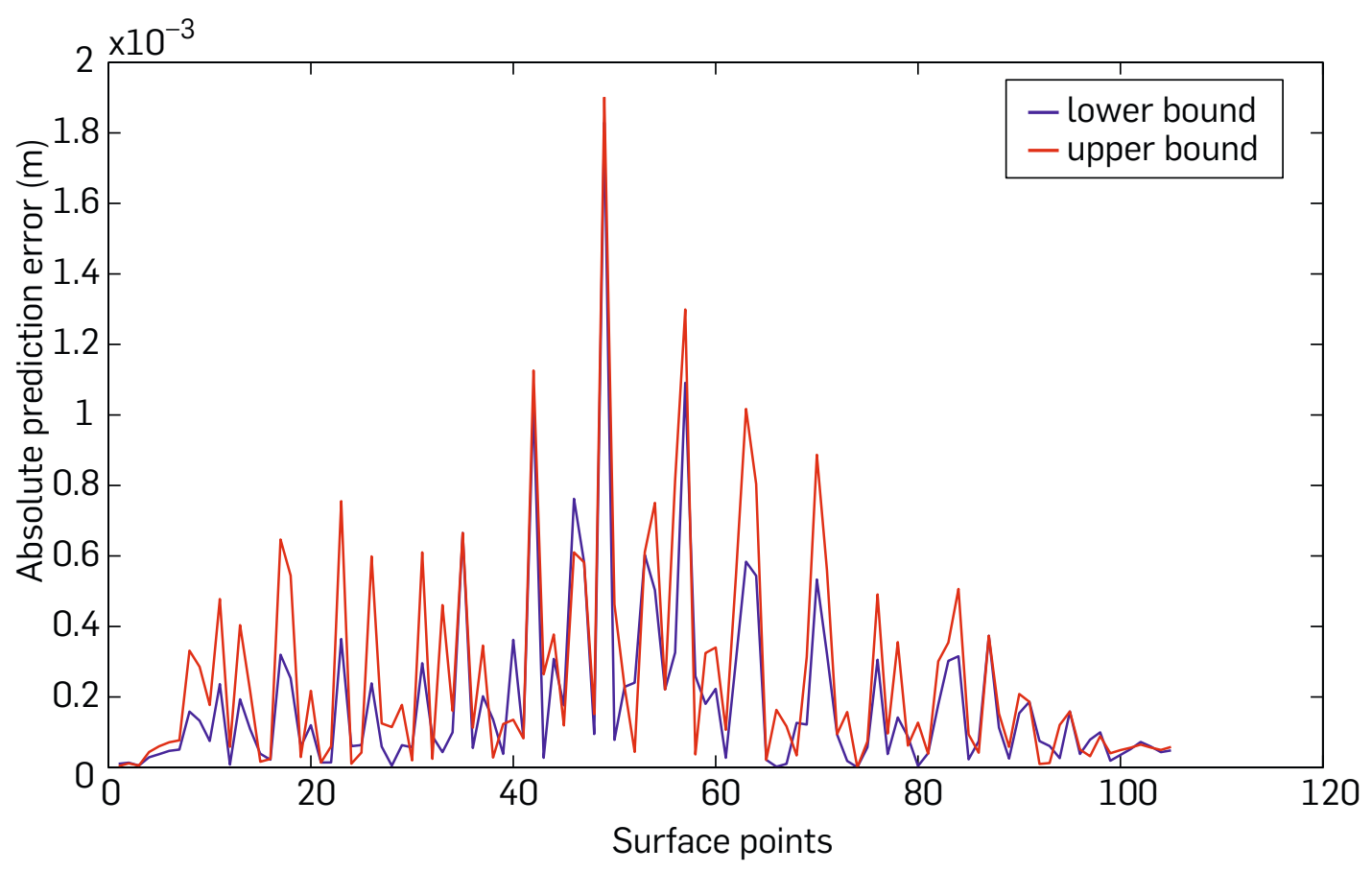

Figure 12: Absolute prediction error of the lower and upper interval settlement bounds at 105 points within the investigated area defined in terms of the reference solution [27]

calculated by

$$
\text { error }=\sqrt{\frac{\sum_{i=1}^{N}\left(\mathbf{S}_{i}^{\text {opt }}-\mathbf{S}_{i}^{*}\right)^{2}}{\sum_{i=1}^{N}\left(\mathbf{S}_{i}^{\text {opt }}\right)^{2}}} \times 100 \%,
$$

are $6.2 \%$ and $8.9 \%$ for the lower and upper bounds, respectively. The largest absolute error is just $1.9 \mathrm{~mm}$, which shows that the prediction capability is appropriate to be used for practical applications. Additionally, the interval bounds computed by the optimisation approach are compared with results of a Monte Carlo simulation, as e.g. applied in [74]. Here, an analysis with a Monte Carlo simulation of $10^{5}$ samples together with the deterministic surrogate model described in [27] was carried out. The interval results obtained from the Monte Carlo simulation have shown a very good agreement with the one from the optimisation approach. The difference of the lower and upper interval bounds of the settlements computed from the two approaches was recorded less than $1 \%$ for the given interval modulus of elasticity of soil layer 1 $\bar{E}_{1}=[45,52] \mathrm{MPa}$ and several grouting pressure scenarios. This comparison confirms the reliability of the proposed surrogate model approach for practical applications.

In Figure 13, the computed interval settlement field is represented by its lower and upper bounds. The most important benefit of the proposed approach is that the computation time is significantly reduced compared to the optimisation approach. To obtain the interval bounds from the optimisation approach, the required computation time is around 1.5 hours. Meanwhile the proposed approach needs only 4 seconds to 


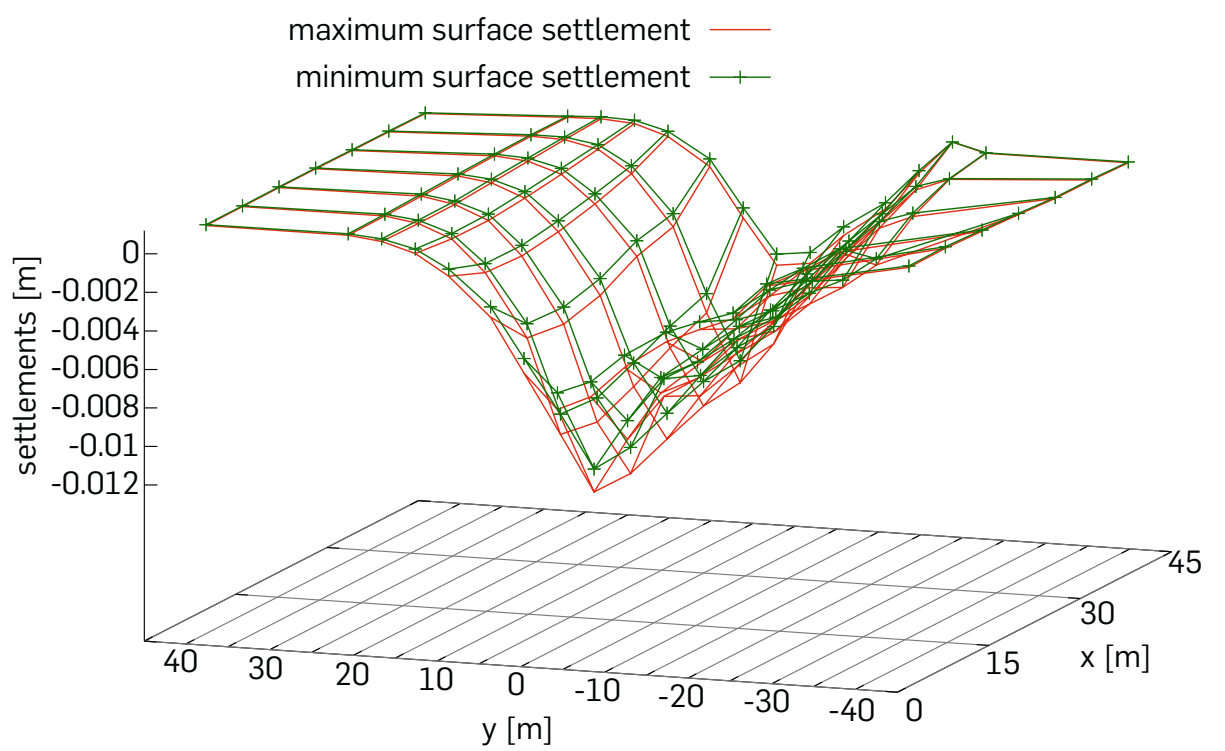

Figure 13: Interval field of tunnelling induced surface settlements with $\bar{E}_{1}=$ $[45,52] \mathrm{MPa}$.

predict the interval settlement field with similar accuracy.

\section{Conclusions}

In the paper, a hybrid surrogate modelling strategy based on Recurrent neural Networks and Proper Orthogonal Decomposition Approaches has been developed to predict time variant settlement fields induced by mechanised tunnelling. The proposed method allows to predict the surface settlements during the tunnel construction in real time for selected scenarios of the TBM steering parameters (e.g. the face and the tail void pressure), taking uncertain geotechnical parameters into account. The hybrid surrogate model parts are created (i.e. trained and tested) by finite element analyses using a process-oriented numerical simulation model taking all relevant components of a tunnel construction process (soil and groundwater conditions, tunnel lining, the TBM with shield and hydraulic jacks, tail void grouting and face support) and their interactions into account.

The uncertainty of geotechnical parameters is quantified by intervals, which are provided as ranges in the geotechnical reports of tunnelling projects. In order to achieve real-time prediction capabilities with uncertain geotechnical parameters, the hybrid RNN-GPOD surrogate model for deterministic data, which has been previously developed by the authors, has been extended to process interval data by means of the midpoint-radius representation. In comparison to the optimisation approach for interval analysis, the computational time is significantly reduced by the proposed strategy. This has been illustrated in the application example, for which the optimisation approach required 1.5 hours while the new approach based on the midpoint-radius 
representation required only 4 seconds to compute the interval bounds of a settlement field with 105 settlement components with a similar accuracy. With such a fast response, the proposed strategy can by utilized for real-time predictions to support the machine driver in steering the TBM, as it enables to quickly investigate the consequences of certain process parameters on the expected settlements in the subsequent excavation stages.

Future developments of the proposed approach include the implementation of further sub-models into the planned TBM steering assistant system, e.g. considering reliability and risk based measures such as tunnel face stability, the risk of damage of the tunnel lining and the damage of existing buildings. In addition, more advanced scenarios with multiple uncertain parameters will be considered. In this case, the number of training samples has to be increased to guarantee a good accuracy of the hybrid surrogate model. Currently, a strategy is developed, which allows to reduce the initial epistemic uncertainty of the geotechnical parameters, i.e. the range of the computed settlements, by updating the resulting interval settlement field by means of deterministic monitoring data measured during the tunnel construction process.

\section{Acknowledgements}

Financial support was provided by the German Research Foundation (DFG) in the framework of project C1 of the Collaborative Research Center SFB 837 "Interaction Modeling in Mechanised Tunnelling". This support is gratefully acknowledged.

\section{References}

[1] B. Maidl, M. Herrenknecht, U. Maidl, G. Wehrmeyer, Mechanised shield tunnelling, Ernst und Sohn, 2013.

[2] G. Swoboda, Abu-Krisha, "Three-dimensional numerical modelling for TBM tunnelling in consolidated clay", Tunnelling and Underground Space Technology, 14: 327-333, 1999.

[3] K. Komiya, "FE modelling of excavation and operation of a shield tunnelling machine", Geomechanics and Tunneling, 2(2): 199-208, 2009.

[4] N. Do, D. Dias, P. Oreste, I. Djeran-Maigre, “Three-dimensional numerical simulation for mechanized tunnelling in soft ground: the influence of the joint pattern", Acta Geotechnica, 9(4): 673-694, 2014, ISSN 1861-1125, URL http://dx.doi.org/10.1007/s11440-013-0279-7.

[5] T. Kasper, G. Meschke, "A 3D finite element model for TBM tunneling in soft ground", International Journal for Numerical and Analytical Methods in Geomechanics, 28: 1441-1460, 2004. 
[6] G. Meschke, J. Ninic, J. Stascheit, A. Alsahly, "Parallelized Computational Modeling of Pile-Soil Interactions in Mechanized Tunneling", Engineering Structures, 47: 35 - 44, 2013, Invited paper for Special Issue Computational Mechanics.

[7] A. Alsahly, J. Stascheit, G. Meschke, "Computational Framework for 3D Adaptive Simulation of Excavation and Advancement Processes in Mechanized Tunneling", in G. Meschke, J. Eberhardsteiner, K. Soga, T. Schanz, M. Thewes (Editors), Computational Methods in Tunneling and Subsurface Engineering (EURO:TUN 2013), pages 85-96, 2013.

[8] S. Freitag, M. Beer, K. Phoon, J. Stascheit, J. Ninić, B. Cao, G. Meschke, "Concepts for Reliability Analyses in Mechanised Tunnelling - Part 1: Theory", in G. Meschke, J. Eberhardsteiner, T. Schanz, K. Soga, M. Thewes (Editors), Proceedings of the Third International Conference on Computational Methods in Tunnelling and Subsurface Engineering (EURO:TUN 2013), pages 791-799. Aedificatio Publishers, Bochum, 2013.

[9] J. Stascheit, S. Freitag, M. Beer, K. Phoon, J. Ninić, B. Cao, G. Meschke, "Concepts for Reliability Analyses in Mechanised Tunnelling - Part 2: Application", in G. Meschke, J. Eberhardsteiner, T. Schanz, K. Soga, M. Thewes (Editors), Proceedings of the Third International Conference on Computational Methods in Tunnelling and Subsurface Engineering (EURO:TUN 2013), pages 801-811. Aedificatio Publishers, Bochum, 2013.

[10] H. Bui, G. Meschke, "Study on Performance of Parallel Solvers for Coupled Simulations of Partially Saturated Soils in Tunnel Engineering Application", in PARENG 2015, 2015.

[11] I. Papaioannou, H. Heidkamp, A. Duester, E. Rank, C. Katz., "Random field reliability analysis as a means for risk assessment in tunnelling", in G. Meschke, G. Beer, J. Eberhardsteiner, D. Hartmann, M. Thewes (Editors), Proceedings of the 2nd International Conference on Computational Methods in Tunnelling (EURO:TUN 2009), pages 585-592. Ruhr-University Bochum, Taylor and Francis, 2009.

[12] K. Phoon, F. Kulhawy, "Characterization of geotechnical variability", Canadian Geotechnical Journal, 36: 612-624, 1999.

[13] B. Möller, M. Beer, "Engineering computation under uncertainty - Capabilities of non-traditional models", Computers and Structures, 86: 1024-1041, 2008.

[14] Z. Bieniawski, Engineering Rock Mass Classifications, Wiley, New York, 1989.

[15] T. Fetz, M. Oberguggenberger, J. Jäger, D. Köll, G. Krenn, H. Lessmann, R. Stark, "Fuzzy Models in Geotechnical Engineering and Construction Management", Computer-Aided Civil and Infrastructure Engineering, 14: 93-106, 1999. 
[16] M. Beer, Y. Zhang, S. Quek, K. Phoon, "Reliability analysis with scare information: Comparing alternative approaches in a geotechnical engineering context", Structural Safety, 41: 1-10, 2013.

[17] A. Nasekhian, H. Schweiger, "Random set finite element method application to tunnelling", International Journal for Reliability and Safety, 5: 299-319, 2011.

[18] J. Kleijnen, "Regression metamodels for generalizing simulation results", IEEE Transactions on Systems, Man, and Cybernetics, 9(2): 93 - 96, 1979.

[19] H. Adeli, "Neural Networks in Civil Engineering: 1989-2000", Computer-Aided Civil and Infrastructure Engineering, 16: 126-142, 2001.

[20] S. Freitag, "Artificial Neural Networks in Structural Mechanics", in Y. Tsompanakis, J. Kruis, B. Topping (Editors), Computational Technology Reviews, Volume 12, pages 1-26. Saxe-Coburg Publications, Stirlingshire, 2015.

[21] J. Kleijnen, Design and Analysis of Simulation Experiments, Springer Science + Business Media, 2008.

[22] B. Ankenman, B.L. Nelson, J. Staum, "Stochastic Kriging for Simulation Metamodeling", Oerations Reserach, 58(2): 371 - 382, 2010.

[23] J.P. Kleijnen, R.G. Sargent, "A Methodology for Fitting and Validating Metamodels in Simulation", European Journal of Operational Research, 120: 14 $29,2000$.

[24] V. Buljak, G. Maier, "Proper Orthogonal Decomposition and Radial Basis Functions in material characterization based on instrumented indentation", Engineering Structures, 33: 492-501, 2011.

[25] K. Khaledi, S. Miro, M. König, T. Schanz, "Robust and reliable metamodels for mechanized tunnel simulations", Computers and Geotechnics, 61: 1-12, 2014.

[26] J. Ninić, G. Meschke, "Model update and real-time steering of tunnel boring machines using simulation-based meta models", Tunnelling and Underground Space Technology, 45: 138-152, 2015.

[27] S. Freitag, B.T. Cao, J. Ninić, G. Meschke, "Hybrid surrogate modelling for mechanised tunnelling simulations with uncertain data", International Journal of Reliability and Safety, 9(2/3): 154-173, 2015, Special Issue on Reliability and Computations of Infrastructures.

[28] S. Freitag, B. Cao, J. Ninić, G. Meschke, "Hybrid RNN-GPOD Surrogate Model for Simulation and Monitoring Supported TBM Steering", in Y. Tsompanakis, J. Kruis, B. Topping (Editors), Proceedings of the 4th International Conference on Soft Computing Technology in Civil, Structural and Environmental Engineering (CSC2015), pages Paper 2, DOI: 10.4203/ccp.109.2. Civil-Comp Press, Prague, 2015. 
[29] F. Nagel, J. Stascheit, G. Meschke, "Process-oriented numerical simulation of shield tunneling in soft soils", Geomechanics and Tunnelling, 3(3): 268-282, 2010 .

[30] P. Dadvand, R. Rossi, E. Oñate, "An Object-oriented Environment for Developing Finite Element Codes for Multi-disciplinary Applications", Archives of Computational Methods in Engineering, 17: 253-297, 2010.

[31] H. Yu, "CASM: A unified state parameter model for clay and sand", International Journal for Numerical and Analytical Methods in Geomechanics, 48: 773-778, 1998.

[32] G. Meschke, C. Kropik, H. Mang, "Numerical analyses of tunnel linings by means of a viscoplastic material model for shotcrete", International Journal for Numerical Methods in Engineering, 39: 3145-3162, 1996.

[33] S. Schindler, P. Mark, "Evaluation of Building Stiffness in the Risk-assessment of Structures Affected by Settlements.", in Proc. 3rd Int. Conf. on Comp. Meth. in Tunneling and Subsurface Engineering - EURO:TUN 2013, RUB, pages 477486. Bochum, Germany, 2013.

[34] S. Schindler, F. Hegemann, A. Alsahly, T. Barciaga, M. Galli, C. Koch, K. Lehner, "Eine Interaktionsplattform für maschinelle Tunnelvortriebe: Anwendung am Beispiel der Wehrhahn-Linie in Düsseldorf", Geomechanics and Tunnelling, 2014.

[35] S. Freitag, R.L. Muhanna, W. Graf, "A Particle Swarm Optimization Approach for Training Artificial Neural Networks with Uncertain Data", in M. Vořechovský, V. Sadílek, S. Seitl, V. Veselý, R.L. Muhanna, R.L. Mullen (Editors), Proceedings of the 5th International Conference on Reliable Engineering Computing (REC 2012), pages 151-170. Litera, Brno, 2012.

[36] S. Freitag, W. Graf, M. Kaliske, "Recurrent Neural Networks for Fuzzy Data", Integrated Computer-Aided Engineering, 18(3): 265-280, 2011, Special Issue Data Mining in Engineering.

[37] B. Möller, W. Graf, M. Beer, "Fuzzy structural analysis using $\alpha$-level optimization", Computational Mechanics, 26: 547-565, 2000.

[38] R. Moore, Methods and Applications of Interval Analysis, Volume 2, SIAM, Studies in Applied Mathematics, Philadelphia, 1979.

[39] T.W. Simpson, J.D. Peplinski, P.N. Koch, J.K. Allen, "Metamodels for Computer-based Engineering Design: Survey and recommendations.", Engineering with Computers, 17: 129-150, 2001.

[40] S. Haykin, Neural Networks - A Comprehensive Foundation, Prentice-Hall, Upper Saddle River, 1999. 
[41] J. Ninić, J. Stascheit, G. Meschke, "Prediction of Tunnelling Induced Settlements using Simulation-Based Artificial Neural Networks", in Y. Tsompanakis, B. Topping (Editors), Proceedings of the Second International Conference on Soft Computing Technology in Civil, Structural and Environmental Engineering, page paper 26. Civil-Comp Press, Stirlingshire, Chania, Greece, 2011, CDROM.

[42] J. Ninic, J. Stascheit, G. Meschke, "Simulation-based Steering for Mechanized Tunneling Using an ANN-PSO-Based Meta-Model", in Y. Tsompanakis (Editor), The Third International Conference on Soft Computing Technology in Civil, Structural and Environmental Engineering, page paper 5. Civil-Comp Press, 2013.

[43] J. Elman, "Finding Structure in Time", Cognitive Science, 14: 179211, 1990.

[44] M. Jordan, "Attractor Dynamics and Parallelism in a Connectionist Sequential Machine", in Proceedings of the eighth annual conference of the cognitive science society. (Amherst 1986), page 531 546, 1986.

[45] A. Chatterjee, "An Introduction to the Proper Orthogonal Decomposition", Current Science, 78: 808-817, 2000.

[46] T. Smith, J. Moehlis, P. Holmes, "Low-Dimensional Modelling of Turbulence Using Proper Orthogonal Decomposition: A Tutorial”, Nonlinear Dynamics, 41: 275-307, 2005.

[47] H. Hotelling, "Analysis of a Complex System of Statistical Variables into Principal Components", Journal of Educational Psychology, 24: 417-441;498-520, 1933.

[48] G. Golub, C. Reinsch, "Singular Value Decomposition and Least Squares Solutions", Numerische Mathematik, 14(5): 403-420, 1970.

[49] K. Karhunen, "Zur Spektraltheorie Stochastischer Prozesse", Ann. Acad. Sci. Fennicae, 37, 1946.

[50] M. Loeve, Probability Theory II, Volume 46 of Graduate Texts in Mathematics, Springer Verlag, New York, 4 edition, 1978.

[51] R. Everson, L. Sivorich, "Karhunen-Loeve Procedure for Gappy Data", Journal of the Optical Society of America A: Optics, Image Science and Vision, 12(8): 1657-1664, 1995.

[52] P. Holmes, J. Lumley, G. Berkooz, Turbulence, Coherent Structures, Dynamical Systems and Symmetry, Cambridge University Press, New York, 1996.

[53] T. Bui-Thanh, M. Damodaran, K. Willcox, "Aerodynamic Data Reconstruction and Inverse Design using Proper Orthogonal Decomposition", The American Institute of Aeronautics and Astronautics (AIAA), 42: 1505-1516, 2004. 
[54] A. Radermacher, S. Reese, "Model Reduction in Elastoplasticity: Proper Orthogonal Decomposition Combined with Adaptive Sub-structuring", Computational Mechanics, 54(3): 677-687, 2014.

[55] F. Galland, A. Gravouil, E. Malvesin, M. Rochette, "A Global Model Reduction Approach for 3D Fatigue Crack Growth with Confined Plasticity", Computer Method in Applied Mechanics and Engineering, 200(5-8): 699-716, 2011.

[56] Z. Ostrowski, R. Bialecki, A. Kassab, "Estimation of Constant Thermal Conductivity by Use of Proper Orthogonal Decomposition”, Comput. Mech., 37: 52-59, 2005.

[57] Z. Ostrowski, R. Bialecki, A. Kassab, "Solving Inverse Heat Conduction Problems Using Trained POD-RBF Network Inverse Method", Inverse Problems in Science and Engineering, 16(1): 39-54, 2008.

[58] K. Khaledi, T. Schanz, S. Miro, "Application of Metamodelling Techniques for Mechanized Tunnel Simulation", Journal of Theoretical and Applied Mechanics, 44(1): 45-54, 2014.

[59] R. Hardy, "Theory and Applications of the Multiquadric-biharmonic Method: 20 Years of Discovery 1968-1988", Computers \& Mathematics with Applications, 19(8-9): 163-208, 1990.

[60] M. Buhmann, Radial Basis Functions, Cam, 2003.

[61] R. Hardy, "Multiquadric Equations of Topography and Other Irregular Surfaces.”, Journal of Geophysical Research, 76: 1905-1915, 1971.

[62] P. Paatero, U. Tapper, "Positive Matrix Factorization: A Nonnegative Factor Model with Optimal Utilization of Error Estimates of Data Values", Environmetrics, 5(2): 111-126, 1994.

[63] P. Paatero, "Least Squares Formulation of Robust Nonnegative Factor Analysis", Chemometrics and Intelligent Laboratory Systems, 37(1): 23-35, 1997.

[64] D. Lee, H. Seung, "Learning the Parts of Objects by Non-Negative Matrix Factorization", Nature, 401(6755): 788-791, 1999.

[65] A. Cichocki, R. Zdunek, A. Phan, S. Amari, Nonnegative Matrix and Tensor Factorizations: Applications to Exploratory Multi-Way Data Analysis and Blind Source Separation, John Wiley \& Sons, 2009.

[66] I. Buciu, "Non-Negative Matrix Factorization, a New Tool for Feature Extraction: Theory and Applications", International Journal of Computers Communications \& Control, 3: 67-74, 2008.

[67] S. Sra, I. Dhillon, "Nonnegative Matrix Approximation: Algorithms and Applications", Technical report, University of Texas, 2006. 
[68] K. Drakakis, S. Rickard, R. de Frein, A. Cichocki, "Analysis of Financial Data Using Non-Negative Matrix Factorization", International Mathematical Forum, 3(38): 1853-1870, 2008.

[69] Y. Wang, Y. Zhang, "Nonnegative Matrix Factorization: A Comprehensive Review", IEEE Transactions on Knowledge and Data Engineering, 25(6): 13361353, 2013.

[70] D. Lee, H. Seung, "Algorithms for Non-Negative Matrix Factorization", in T. Leen, T. Dietterich, V. Tresp (Editors), Advances in Neural Information Processing Systems, pages 556-562, 2001.

[71] H. Kim, H. Park, "Nonnegative Matrix Factorization Based on Alternating Nonnegativity Constrained Least Squares and Active Set Method", SIAM Journal on Matrix Analysis and Applications, 30(2): 713-730, 2008.

[72] M. Van Benthem, M. Keenan, "Fast Algorithm for the Solution of Large-Scale Non-Negativity-Constrained Least Squares Problems", Journal of Chemometrics, 18: 441-450, 2004.

[73] S. Freitag, B.T. Cao, J. Ninić, G. Meschke, "Surrogate modeling for mechanized tunneling simulations with uncertain data", in M. Modares (Editor), Proceedings of the 6th International Workshop on Reliable Engineering Computing (REC 2014), pages 44-63. Chicago, 2014.

[74] E. Patelli, D. Alvarez, M. Broggi, M. de Angelis, "Uncertainty Management in Multidisciplinary Design of Critical Safety Systems", Journal of Aerospace Information Systems, 12(1): 140-169, 2015. 